\title{
Parallel Repetition of Two-Prover One-Round Games: An Exposition
}

\author{
Suguru TAMAKI* \\ Graduate School of Informatics, Kyoto University, Kyoto 606-8501, Japan
}

\begin{abstract}
A two-prover one-round game is a fundamental combinatorial optimization problem arising from such areas as interactive proof systems, hardness of approximation, cryptography and quantum mechanics. The parallel repetition theorem states that for any two-prover one-round game with value smaller than $1, k$-fold parallel repetition reduces the value of the game exponentially in $k$. The theorem was originally proved by Raz (SICOMP 1998) and later simplified and improved by Holenstein (Theory of Computing 2009) and Rao (SICOMP 2011). All the known proofs are based on information theoretic arguments. Very recently, Dinur and Steurer (STOC 2014) obtained a new proof of the parallel repetition theorem based on a matrix analysis argument. In this paper, we describe a special case of Dinur and Steurer's proof. We also describe an application of the parallel repetition theorem to inapproximability results of two-prover one-round games. Our presentation is almost self-contained in the sense that we only assume the PCP theorem. To do so, we also present proofs for the necessary results related to algebraic graph theory and hardness of approximation.
\end{abstract}

KEYWORDS: interactive proof system, hardness of approximation, spectral graph theory, matrix analysis, label cover

\section{Introduction}

In this paper, we are concerned with parallel repetition of two-prover one-round games and its application to hardness of approximation. More specifically, we present a proof of an important special case of the parallel repetition theorem for two-prover one-round games following Dinur and Steurer's matrix analytic approach [17]. We also present a proof of inapproximability results for two-prover one-round games, which serves as a starting point of a lot of optimal inapproximability results.

Before stating the parallel repetition theorem and inapproximability results for two-prover one-round games formally, we briefly review the background on the study of parallel repetition in the next section to motivate readers.

\subsection{Why do we care about parallel repetition?}

The motivation for studying parallel repetition comes from the areas of interactive proof systems and hardness of approximation. For more details on these topics, see standard textbooks on computational complexity, e.g., [2].

\subsubsection{Original motivation: On the power of multi-prover interactive proof systems}

Multi-prover interactive proof systems were introduced by Ben-Or, Goldwasser, Kilian and Wigderson [9]. In a $k$-prover $r$-round interactive proof systems, computationally unbounded provers $P_{1}, P_{2}, \ldots, P_{k}$ try to convince a probabilistic polynomial time verifier $V$ that a common input $x$ satisfies a property $L$, i.e., $x \in L$. The verifier performs a computation at most $r$ rounds following a protocol. In each round, the verifier sends to each prover a query and receives an answer. The protocol specifies how the verifier makes queries based on an input and answers received in previous rounds. The important assumption is that the provers do not communicate with each other during the protocol (otherwise we can merge provers into a single prover). They may communicate before the protocol and agree on a shared strategy to answer queries. When the verifier finishes the final round, it decides whether the input satisfies a property (i.e., accept) or not (i.e., reject).

A couple of remarks are in order. The goal of the provers is to convince $x \in L$ even if $x \notin L$ while the goal of the verifier is to decide correctly whether $x \in L$ or not. Since $V$ is a randomized algorithm, it is natural to allow error in the final decision of $V$ with some probability. Technically we say a protocol has completeness $c \in[0,1]$ if $V$ accepts $x$ with probability at least $c$ for any $x \in L$ and has soundness $s \in[0,1]$ if $V$ accepts $x$ with probability at most $s$ for any $x \notin L$. We denote by $\operatorname{MIP}_{c, s}(k, r)$ the set of properties (languages) for which $k$-prover $r$-round protocols with completeness $c$ 
and soundness $s$ exist.

Interactive proof systems can be regarded as a generalization of nondeterministic polynomial time computation. Recall that the class NP is the set of languages (properties) decidable by nondeterministic polynomial time Turing machines. It follows from the definition that $\mathrm{NP} \subseteq \operatorname{MIP}_{1,0}(1,1)$. For example, let us consider the 3-satisfiability problem (3SAT). In 3SAT, the task is, given a 3CNF formula such as $\phi=\left(x_{1} \vee \bar{x}_{2} \wedge x_{3}\right) \wedge\left(x_{2} \vee x_{4} \vee x_{5}\right) \wedge$ $\left(\bar{x}_{1} \vee \bar{x}_{3} \vee x_{4}\right) \cdots$, to decide whether there exists a satisfying assignment, i.e., $0 / 1$ assignment to the variables $x_{1}, x_{2}, \ldots$ that satisfies all the clauses. The following 1-prover 1-round protocol for 3SAT has $c=1$ and $s=0$ :

(1) The verifier asks the prover a satisfying assignment for the input formula and receives a $0 / 1$ assignment.

(2) The verifier evaluates the assignment and accepts if and only if it satisfies the input.

If we use more provers and rounds and also allow errors, i.e., set $c<1$ and/or $s>0$, the power of protocols is likely to become strong, and this is actually the case. $\operatorname{MIP}_{1,1 / 3}(1,1)$ contains the graph non-isomorphism problem, which is not known to be in NP. Lund, Fortnow, Karlof and Nisan [37] and Shamir [46] showed that $\operatorname{MIP}_{1,1 / 3}(1, \operatorname{poly}(n))$ is exactly the same set as PSPACE which is the set of languages decidable by polynomial space Turing machines. Here $\operatorname{MIP}_{1,1 / 3}(1, \operatorname{poly}(n))$ means that the number of rounds can be polynomially long with respect to input length $n$. Babai, Fortnow and Lund [5] showed that $\operatorname{MIP}_{1,2^{-n}}(2, \operatorname{poly}(n))$ is exactly the same set as NEXP which is the set of languages decidable by nondeterministic exponential time Turing machines. Lapidot and Shamir [35] proved that $\operatorname{MIP}_{1,2^{-n}}(4,1)=$ NEXP. Feige [20] proved that for some $\delta>0, \operatorname{MIP}_{1,1-\delta}(2,1)=$ NEXP. This result is a strengthening of $\operatorname{MIP}_{1,2^{-n}}(2, \operatorname{poly}(n))=$ NEXP because if we repeat the protocol for NEXP by Feige sequentially $O(n)$ times, we obtain a protocol with polynomial rounds and exponentially small soundness.

From the results explained above, it is natural to ask whether $\operatorname{MIP}_{1,2-n}(2,1)=$ NEXP or not. One possible approach to resolve this question affirmatively is parallel repetition of protocols. What happens if the verifier executes the oneround protocol of Feige $n$ times in parallel? One might hope that if the soundness of the original protocol is $s$, the soundness of the parallel-repeated protocol is $s^{n}$. Unfortunately, this is not true, but we can still hope that the soundness might be exponentially small in $s$, i.e., $\left(s^{\prime}\right)^{n}$ for some $s^{\prime}$ which depends only on $s$. This is the parallel repetition conjecture posed by Feige and Lovász [24]. Since any two-prover one-round interactive proof system can be viewed as a two-prover one-round game between the verifier and the two provers, Feige and Lovász formulated the conjecture as an effect of a certain operation on such games; see Section 1.2 for the precise definition. We remark that Feige and Lovász showed that $\operatorname{MIP}_{1,2^{-n}}(2,1)=\mathrm{NEXP}$ without proving the conjecture. The conjecture was finally resolved by Raz [42].

Such two-prover one-round games also arise in cryptographic applications [9, 10,19,34] and hardness of approximation results $[3,4,22,24,38]$, and have been used to prove direct product theorems for communication complexity [40]. We elaborate on the connection to hardness of approximation in the next section.

\subsubsection{Applications: Optimal inapproximability results}

As we saw in the previous section, the original motivation for studying parallel repetition comes from the question about structural complexity theory. However, after the parallel repetition conjecture became the parallel repetition theorem due to Raz, the theorem has been most often used to show the limitation of approximation algorithms.

Approximation algorithms have been studied to cope with computationally intractable (i.e., NP-hard) optimization problems. For example, in the maximum 3-satisfiability problem (Max 3SAT), the task is, given a 3CNF formula, to find an assignment that maximizes the number of satisfied clauses. Of course this is an NP-hard problem and we cannot expect polynomial time algorithms for it. We say a polynomial time algorithm is $\rho$-approximation $(\rho<1)$ if the algorithm always finds an assignment that satisfies $\rho$ times as many clauses as the best assignment does. It is easy to give a 7/8-approximation algorithm: the algorithm just picks an assignment uniformly at random. Can we do better? The possibility that we can design a $\rho$-approximation algorithm with $\rho$ arbitrary close to 1 was ruled out by the famous PCP theorem $[3,4]$. Namely, it states that there exists some $\delta>0$ such that if a $(1-\delta)$-approximation algorithm exists, then $\mathrm{P}=\mathrm{NP}$. However, $\delta$ above is a tiny constant and there is a gap between $(1-\delta)$ and $7 / 8$, so we might obtain a $(7 / 8+0.01)$-approximation algorithm. Surprisingly, Håstad [26] proved that $7 / 8$-approximation is best possible in the sense that for any $\varepsilon>0$, if a $(7 / 8+\varepsilon)$-approximation algorithm exists, then $\mathrm{P}=\mathrm{NP}$.

The parallel repetition theorem plays an important role in Håstad's proof of the above result. He applied the theorem for two-prover one-round games to obtain hard-to-approximate two-prover one-round games. Then he combined it with a sophisticated gadget reduction (called Long Code) to Max 3SAT. The reduction guarantees that if a $(7 / 8+$ $\varepsilon)$-approximation algorithm exists, then there exists a "good" approximation algorithm for two-prover one-round games.

\subsection{Formal statement of parallel repetition theorem}

A two-prover one-round game $G$ (often called game in this paper for short) consists of a bipartite graph with vertex sets $U, V$, an edge set $E \subseteq U \times V$ and a label set $\Sigma$. Also each edge $e \in E$ has a positive weight $w_{e}$ and a constraint $\pi_{e} \subseteq \Sigma \times \Sigma$. We can represent a game as $G=(U, V, E, \Sigma, W, \Pi)$ where $W=\left\{w_{e}\right\}_{e \in E}$ and $\Pi=\left\{\pi_{e}\right\}_{e \in E}$. The constraint graph of $G$ is the graph obtained by just ignoring $\Sigma$, $\Pi$ of $G$. We say assignments $f: U \rightarrow \Sigma$ and $g: V \rightarrow \Sigma$ satisfy $\pi_{(u, v)}$ if $(f(u), g(v)) \in \pi_{(u, v)}$ holds. In the LabelCover problem, our goal is to find assignments $f: U \rightarrow \Sigma$ and 
$g: V \rightarrow \Sigma$ that maximize the value of the game, i.e., the normalized sum of the weights of satisfied edges,

$$
\operatorname{val}(G):=\max _{f, g} \underset{(u, v) \sim E}{\mathbf{P}}\left\{(f(u), g(v)) \in \pi_{(u, v)}\right\}=\max _{f, g} \frac{1}{\sum_{e \in E} w_{e}} \times \sum_{(u, v) \in E:(f(u), g(v)) \in \pi_{(u, v)}} w_{(u, v)},
$$

where the maximum is taken over all the assignments and $\mathbf{P}_{(u, v) \sim E}\left\{(f(u), g(v)) \in \pi_{(u, v)}\right\}$ means the probability that a random edge $(u, v)$ is satisfied by $f, g$ if we sample an edge $(u, v)$ with probability proportional to $w_{(u, v)}$.

Parallel repetition is a basic operation of games defined using tensor product as follows.

Definition 1.1 (Tensor product and parallel repetition).

Let $G=\{U, V, E, \Sigma, W, \Pi\}, G^{\prime}=\left\{U^{\prime}, V^{\prime}, E^{\prime}, \Sigma^{\prime}, W^{\prime}, \Pi^{\prime}\right\}$ be two games. Then tensor product of $G$ and $G^{\prime}$, denoted by $G \otimes G^{\prime}$, is defined as a bipartite graph with

- vertex sets $U \times U^{\prime}, V \times V^{\prime}$,

- an edge set $E \otimes E^{\prime}:=\left\{\left(u, u^{\prime}, v, v^{\prime}\right) \mid(u, v) \in E \wedge\left(u^{\prime}, v^{\prime}\right) \in E^{\prime}\right\}$,

- a label set $\Sigma \times \Sigma^{\prime}$,

- each edge $\left(u, u^{\prime}, v, v^{\prime}\right)$ has a weight $w_{(u, v)} \cdot w_{\left(u^{\prime}, v^{\prime}\right)}^{\prime}$, and

- each edge $\left(u, u^{\prime}, v, v^{\prime}\right)$ has a constraint

$$
\left\{(a, b, c, d) \mid(a, c) \in \pi_{(u, v)} \wedge(b, d) \in \pi_{\left(u^{\prime}, v^{\prime}\right)}^{\prime}\right\} \subseteq\left(\Sigma \times \Sigma^{\prime}\right) \times\left(\Sigma \times \Sigma^{\prime}\right) .
$$

Then, $k$-fold parallel repetition of $G$, denoted by $G^{\otimes k}$, is defined inductively as $G^{\otimes 1}:=G, G^{\otimes k}:=G \otimes G^{\otimes(k-1)}$. We are interested in the relation between $\operatorname{val}(G)$ and $\operatorname{val}\left(G^{\otimes k}\right)$. It is easy to see that $\operatorname{val}\left(G^{\otimes k}\right) \geq \operatorname{val}(G)^{k}$. However, upper bounding $\operatorname{val}\left(G^{\otimes k}\right)$ as a function of $\operatorname{val}(G)$ is highly non-trivial.

Example 1.2.

$$
\begin{aligned}
U & =\{1,2\}, V=\{3,4\}, E=\{(1,3),(1,4),(2,3),(2,4)\}, \Sigma=\{1,2,3,4\}, \\
w_{(u, v)} & =1 \text { for every }(u, v) \in E \\
\pi_{(u, v)} & =\{(u, u),(v, v)\} \text { for each }(u, v) \in E .
\end{aligned}
$$

One can see that $\operatorname{val}(G)=1 / 2$, e.g., $f(1)=f(2)=g(3)=g(4)=1$ is one of the optimal assignments. Surprisingly, we can show that $\operatorname{val}\left(G^{\otimes 2}\right)=1 / 2$ with the following assignments:

$$
\begin{aligned}
& f(1,1)=f(1,2)=(1,3), f(2,1)=f(2,2)=(2,4), \\
& g(3,3)=g(4,3)=(1,3), g(3,4)=g(4,4)=(2,4) .
\end{aligned}
$$

The above example shows that $k$-fold parallel repetition does not necessarily decrease the game value for small $k$. However, for sufficiently large $k, \operatorname{val}\left(G^{\otimes k}\right)$ approaches to 0 . The first upper bound on $\operatorname{val}\left(G^{\otimes k}\right)$ was obtained by Verbitsky.

Theorem 1.3 ([51]). If $\operatorname{val}(G)<1$, then $\lim _{k \rightarrow \infty} \operatorname{val}\left(G^{\otimes k}\right)=0$.

Various papers give upper bounds on the effect of $k$-fold parallel repetition [12, 20, 34, 51] but all of them fall short to give a result resolving the parallel repetition conjecture. Raz then gave a very strong result, that is, the following:

Theorem 1.4 (Parallel Repetition Theorem [42]). For every $\varepsilon>0$ and $\sigma$, there exists a constant $\tau_{\varepsilon, \sigma}<1$ such that for a game $G$ with $\operatorname{val}(G)<1-\varepsilon$ and $|\Sigma|=\sigma, \operatorname{val}\left(G^{\otimes k}\right) \leq\left(\tau_{\varepsilon, \sigma}\right)^{k}$ holds.

It is the only explicit bound for games with arbitrary weight distributions and quantitatively the strongest until recently. We give a brief overview of known bounds in Section 1.3. We remark that Theorem 1.3 does not imply Theorem 1.4 since we cannot set $\tau_{\varepsilon, \sigma}$ independently of $k$ and the size of $G$ in Theorem 1.3.

Theorem 1.4 has a strong application in hardness of approximation. We denote by LabelCover $(1, \delta)$ the promise problem* of distinguishing the following two cases given a game $G$ : (yes case) $\operatorname{val}(G)=1$, or (no case) $\operatorname{val}(G) \leq \delta$. The combination of Theorem 1.4 and the PCP theorem [3,4] yields the following.

Theorem 1.5. For any $\delta>0$, there exists a constant $\sigma$ such that LabelCover $(1, \delta)$ over regular projection games with the alphabet size $\sigma=|\Sigma|$ is NP-hard.

Here we say a game $G$ is regular if the underlying graph of $G$ is bipartite and satisfies some additional condition; see Section 3 for a precise definition. We also say that $G$ is a projection game if every $\pi_{(u, v)}$ is a projection constraint: each $\beta$ has a unique $\alpha$ for which $(\alpha, \beta) \in \pi_{(u, v)}$. Theorem 1.5 is an important ingredient of several optimal inapproximability results such as Max Clique [25], Max 3LIN and Max 3SAT [26] and many other optimization problems; see, e.g., [49].

\subsection{Bounds on parallel repetition of two-prover one-round games}

In this section, we give an overview of known bounds on parallel repetition. They are better than or incomparable to Raz's result. Let us begin with some definitions. We say $G$ is a unique game if every $\pi_{(u, v)}$ is a unique constraint: each $\beta$ has a unique $\alpha$ for which $(\alpha, \beta) \in \pi_{(u, v)}$ and also each $\alpha$ has a unique $\beta$ for which $(\alpha, \beta) \in \pi_{(u, v)}$. We say $G$ is a free game if there are sets of vertex weights $\left\{w_{u}\right\}_{u \in U}$ and $\left\{w_{v}\right\}_{v \in V}$ such that $w_{(u, v)}=w_{u} \cdot w_{v}$ holds for every $(u, v) \in E$. Let

*A promise problem consists of a set of yes instances $\Pi_{Y}$ and a set of no instances $\Pi_{N}$. The task is, given an input instance $I$, to decide whether $I \in \Pi_{Y}$ or $I \in \Pi_{N}$ holds. If $I$ does not belong to $\Pi_{Y} \cup \Pi_{N}$, then the answer can be arbitrary. A promise problem is called a decision problem if every input instance belongs to $\Pi_{Y} \cup \Pi_{N}$. 
$\sigma=|\Sigma|$ be the alphabet size of the underlying game.

Theorem 1.4 can be stated more precisely as follows.

Theorem 1.6 (Raz [42]). There is a constant $\alpha>0$ such that for every game $G$ with value $1-\varepsilon$, the value of $G^{\otimes k}$ is at most $(1-\varepsilon / 2)^{\alpha \varepsilon^{32} k / \log _{2} \sigma}$.

The proof of the above theorem is known as one of the most difficult proofs in theoretical computer science. Holenstein succeeded in simplifying the proof and also improved the bounds as follows.

Theorem 1.7 (Holenstein [27]). There is a constant $\alpha>0$ such that for every game $G$ with value $1-\varepsilon$, the value of $G^{\otimes k}$ is at most $(1-\varepsilon / 2)^{\alpha \varepsilon^{2} k / \log _{2} \sigma}$.

Rao gave an even better bound for a special case of projection games.

Theorem 1.8 (Rao [41]). There is a constant $\alpha>0$ such that for every projection game $G$ with value $1-\varepsilon$, the value of $G^{\otimes k}$ is at most $(1-\varepsilon / 2)^{\alpha \varepsilon k}$.

All the above three theorems are proved by information theoretic arguments.

In contrast, Dinur and Steurer took a different approch, i.e., matrix analysis approach to prove the parallel repetition theorem and obtained the following results.

Theorem 1.9 (Dinur and Steurer [17]). For every regular projection game $G$ with value $\rho$, the value of $G^{\otimes k}$ is at most $(\sqrt{2 \rho})^{k}$.

Theorem 1.10 (Dinur and Steurer [17]). For every regular projection game $G$ with value $1-\varepsilon$, the value of $G^{\otimes k}$ is at most $1-\Omega(\sqrt{k} \cdot \varepsilon)$ for $k \ll 1 / \varepsilon^{2}$.

Theorem 1.11 (Dinur and Steurer [17]). Let $G_{1}, \ldots, G_{k}$ be regular projection games. Then

$$
\operatorname{val}\left(G_{1} \otimes \cdots \otimes G_{k}\right) \leq \prod_{i=1}^{k} \frac{2 \sqrt{\delta_{i}}}{1+\delta_{i}}
$$

where $\delta_{i}=\operatorname{val}\left(G_{i}\right)$.

Proofs of these bounds using information theoretic arguments are not known. Theorem 1.9 combined with [15, 39] implies the following inapproximability result.

Theorem 1.12 (Dinur and Steurer [17]). For every constant a $>0$, given a Label Cover instance of size $n$ with alphabet size at most $n$, it is NP-hard to decide if its value is 1 or at most $\varepsilon=\frac{1}{(\log n)^{a}}$.

This hardness result enables us to establish optimal inapproximability results for the set cover problem under the assumption that $\mathrm{P} \neq \mathrm{NP}$; see [17].

If we restrict input instances to free games or games on expander graphs, we have stronger bounds summarized as follows. Here we call a graph expander if its random walk matrix has a constant spectral gap; see Section 2 for the precise definitions of random walk matrix and spectral gap.

Theorem 1.13 (Barak et al. [8]). There is a constant $\alpha>0$ such that for every free game $G$ with value $1-\varepsilon$ for $\varepsilon<1 / 2$, the value of $G^{\otimes k}$ is at most $\left(1-\varepsilon^{2}\right)^{\alpha k / \log _{2} \sigma}$.

Theorem 1.14 (Barak et al. [8]). There is a constant $\alpha>0$ such that for every free projection game $G$ with value $1-\varepsilon$ for $\varepsilon<1 / 2$, the value of $G^{\otimes k}$ is at most $(1-\varepsilon)^{\alpha k}$.

Let $\gamma$ be the spectral gap of the random walk matrix of the constraint graph. ${ }^{\dagger}$

Theorem 1.15 (Raz and Rosen [45]). There is a constant $\alpha>0$ such that for every game $G$ with value $1-\varepsilon$ for $\varepsilon<1 / 2$, the value of $G^{\otimes k}$ is at most $\left(1-\varepsilon^{2} \cdot \operatorname{poly}(\gamma)\right)^{\alpha k / \log _{2} \sigma}$.

Theorem 1.16 (Raz and Rosen [45]). For every projection game $G$ with value $1-\varepsilon$ for $\varepsilon<1 / 2$, the value of $G^{\otimes k}$ is at most $(1-\varepsilon)^{\operatorname{poly}(\gamma) \cdot k}$.

So far we have seen upper bounds on paraller repetition. There are also a few lower bounds known for some special classes of games. For a special case of unique games, called odd cycle games, the following bound was proved.

Theorem 1.17 (Raz [44]). For an odd cycle game $G$ with value $1-1 / m$, the value of $G^{\otimes k}$ is at least $1-(1 / m) \cdot \sqrt{k}$. If $k=\Omega\left(m^{2}\right)$, the value of $G^{\otimes k}$ is at least $\left(1-1 / 4 m^{2}\right)^{O(k)}$.

This theorem means that Theorem 1.8 is essentially tight. The theorem was extended to unique games by [7,47]. Theorem 1.18 (Steurer [47]). For every unique game $G$ with value $1-\varepsilon$, the value of $G^{\otimes k}$ is at least $1-$ $O(\sqrt{k \varepsilon \log \sigma})$.

Summary of the results above is given in Table 1 .

Remark 1.19. We have seen bounds on parallel repetition for several classes of two-prover one-round games. The study of these classes is motivated mainly by two reasons. One reason is the strong parallel repetition conjecture stating that the value of $G^{\otimes k}$ is at most $(1-\Omega(\varepsilon))^{k}$. The conjecture is true for free projection games (Theorem 1.14) and projection games with spectral gaps (Theorem 1.16), but in general, or even for a special case of unique games, it is false. Another reason is applications to hardness of approximation. The inapproximability results for regular projection games serves as a starting point of a lot of inapproximability results, and to obtain "optimal" inapproximability results, the efficiency of parallel repetition is quite important. Also, unique games are useful in proving inapproximability results due to Khot's unique games conjecture (UGC). UGC states that for any $\delta>0$, there exists a constant $\sigma$ such that

${ }^{\dagger}$ Strictly speaking, the definition of $\gamma$ in [45] is different from ours. Our definition is applicable to arbitrary graphs while theirs is specific to bipartite graphs. 
LabelCover $(1-\delta, \delta)$ over unique games with the alphabet size $\sigma=|\Sigma|$ is NP-hard. Under UGC, we can prove optimal inapproximability results for the maximum cut problem, the minimum vertex cover problem and many other problems for which such results have not been proven using the inapproximability results for projection games; see [31, 32]. Remark 1.20. There are two approaches to the parallel repetition theorem, i.e., information theoretic and matrix analysis approaches. Both have their merits: Information theoretic approach establishes a parallel repetition theorem for wider classes of two-prover one-round games, while matrix analysis approach gives a more efficient parallel repetition theorem for special classes of two-prover one-round games. Matrix approach can, as in Theorem 1.11, also treat tensor product of different games. If we consider a "quantum version" of two-prover one-round games, the situation becomes a bit different: Both of information theoretic [13,28] and matrix analysis [18] approaches showed parallel repetition theorems for different classes of games.

Table 1. Summary of known bounds

\begin{tabular}{ccc}
\hline Upper bounds of the value of $G^{\otimes k}$ & Remark (on $G)$ & Reference \\
\hline$(1-\varepsilon / 2)^{\alpha \varepsilon^{32} k / \log _{2} \sigma}$ & - & Theorem 1.6 [42] \\
$(1-\varepsilon / 2)^{\alpha \varepsilon^{2} k / \log _{2} \sigma}$ & - & Theorem 1.7 [27] \\
$(1-\varepsilon / 2)^{\alpha \varepsilon k}$ & projection & Theorem 1.8 [41] \\
$(\sqrt{2 \rho})^{k}$ & regular, projection, value $\rho$ & Theorem 1.9 [17] \\
$1-\Omega(\sqrt{k} \cdot \varepsilon)$ & $k \ll 1 / \varepsilon^{2}$ & Theorem 1.10 [17] \\
$\left(1-\varepsilon^{2}\right)^{\alpha k / \log _{2} \sigma}$ & free & Theorem 1.13 [8] \\
$(1-\varepsilon)^{\alpha k}$ & free, projection & Theorem 1.14 [8] \\
$\left(1-\varepsilon^{2} \cdot \operatorname{poly}(\gamma)\right)^{\alpha k / \log _{2} \sigma}$ & spectral gap $\gamma$ & Theorem 1.15 [45] \\
$(1-\varepsilon)^{\left.\text {ply }^{2}\right) \cdot k}$ & projection, spectral gap $\gamma$ & Theorem 1.16 [45] \\
\hline Lower bounds of the value of $G^{\otimes k}$ & Remark (on $G)$ & Reference \\
\hline $1-(1 / m) \cdot \sqrt{k}$ & odd cycle, value $1-1 / m$ & Theorem 1.17 [44] \\
$\left(1-1 / 4 m^{2}\right)^{O(k)}$ & odd cycle, value $1-1 / m, k=\Omega\left(m^{2}\right)$ & Theorem 1.17 [44] \\
$1-O(\sqrt{k \varepsilon \log \sigma})$ & unique & Theorem 1.18 [47] \\
\hline
\end{tabular}

\subsection{Contribution and organization of this paper}

The main aim of this paper is to explain the essence of the matrix analysis approach due to Dinur and Stuerer [17] used to prove the parallel repetition theorem for regular projection games (see Theorems 1.9, 1.10, and 1.11). For this, we give a rigorous proof of the parallel repetition theorem for some special case of regular projection games (see Theorem 3.3 in Section 3). While the proof for this special case is relatively simpler than the proofs for Theorems 1.9 , 1.10, and 1.11, it is nontrivial enough to give the flavor of the matrix analysis approach in [17]. In addition, we illustrate the power of the parallel repetition theorem by giving a proof of Theorem 1.5 concerning the LabelCover problem, where Theorem 3.3 and the PCP theorem play crucial roles.

The organization of this paper is as follows. In Section 2, we present basic notion and facts from algebraic graph theory, which are the main technical tools of [17]. In Section 3, we describe the proof of the parallel repetition theorem for a special case of regular projection games due to [17]. In Section 4, we show LabelCover $(1,1-\delta)$ on regular projection games is NP-hard for some small constant $\delta$. This hardness result is combined with parallel repetition to establish Theorem 1.5.

\section{Algebraic Graph Theory}

In this section, we present basic notion and facts from algebraic graph theory. The left side of Cheeger's inequality (Lemma 2.10) will play an important role in the proof of Theorem 3.3 in Section 3.

\subsection{Linear algebra review}

In this section, we quickly review basic facts in linear algebra we will use later. Throughout the paper, we only consider real vectors and matrices. Let $M \in \mathbb{R}^{n \times n}$ be an $n \times n$ matrix. We denote by $M(i, j)$ the $(i, j)$-element of $M$. The transpose of $M$ is denoted by ${ }^{t} M$. We regard a vector $x \in \mathbb{R}^{n}$ as a column vector (an $n \times 1$ matrix). For $x, y \in \mathbb{R}^{n}$, the inner product of $x, y$ is defined as $\langle x, y\rangle:=\sum_{i=1}^{n} x(i) y(i)$ and the norm of $x$ is defined as $\|x\|:=\langle x, x\rangle^{1 / 2}$.

Definition 2.1 (Eigenvalue and eigenvector). Let $M$ be an $n \times n$ matrix. Suppose that $M x=\lambda x$ for $x \in \mathbb{R}^{n}, x \neq 0$, and $\lambda \in \mathbb{R}$. Then we call $x$ an eigenvector and $\lambda$ an eigenvalue of $M$.

Proposition 2.2. Suppose $M$ is an $n \times n$ symmetric matrix. Then, the following properties hold:

- If $v$ and $w$ are eigenvectors of $M$ with different eigenvalues, then $v$ and $w$ are orthogonal, i.e., $\langle v, w\rangle=0$.

- If $v$ and $w$ are eigenvectors of $M$ with the same eigenvalue, then so is $q=a v+b w$ for any $a, b \in \mathbb{R}$, so 
eigenvectors with the same eigenvalue need not be orthogonal.

- $M$ has a full orthonormal basis of eigenvectors $v_{1}, \ldots, v_{n}$. All eigenvalues and eigenvectors are real.

- $M$ is diagonalizable:

$$
M={ }^{t} V \Lambda V
$$

where $V$ is orthogonal $\left({ }^{t} V V\right.$ is the identity matrix $\left.I_{n}\right)$, with columns equal to $v_{1}, \ldots, v_{n}$, and $\Lambda$ is diagonal, with the corresponding eigenvalues of $M$ as its diagonal entries. We have $M=\sum_{i=1}^{n} \lambda_{i} v_{i}{ }^{t} v_{i}$.

In Proposition 2.2, it is important that $M$ is symmetric. No results stated there are necessarily true in the case that $M$ is not symmetric.

Definition 2.3. We call the span of the eigenvectors with the same eigenvalue an eigenspace.

Courant-Fischer theorem (Theorem 2.4) gives a variational formulation of the eigenvalues of a symmetric matrix, which can be useful for obtaining bounds on the eigevalues.

Theorem 2.4 (Courant-Fischer Formula). Let $M$ be an $n \times n$ symmetric matrix with eigenvalues $\lambda_{1} \geq \lambda_{2} \geq \cdots \geq \lambda_{n}$ and corresponding eigenvectors $v_{1}, \ldots, v_{n}$. Then

$$
\begin{aligned}
& \lambda_{1}=\max _{\|x\|=1}\langle x, M x\rangle=\max _{x \neq 0} \frac{\langle x, M x\rangle}{\langle x, x\rangle}, \\
& \lambda_{2}=\max _{\|x\|=1, x \perp v_{1}}\langle x, M x\rangle=\max _{x \neq 0, x \perp v_{1}} \frac{\langle x, M x\rangle}{\langle x, x\rangle}, \\
& \lambda_{n}=\min _{\|x\|=1}\langle x, M x\rangle=\min _{x \neq 0} \frac{\langle x, M x\rangle}{\langle x, x\rangle} .
\end{aligned}
$$

In general, for $1 \leq k \leq n$, let $S_{k}$ denote the span of $v_{1}, \ldots, v_{k}$ (with $S_{0}=\{0\}$ ), and let $S_{k}^{\perp}$ denote the orthogonal complement of $S_{k}$. Then

$$
\lambda_{k}=\max _{\|x\|=1, x \in S_{k-1}^{\perp}}\langle x, M x\rangle=\max _{x \neq 0, x \in S_{k-1}^{\perp}} \frac{\langle x, M x\rangle}{\langle x, x\rangle} .
$$

Proof. Let $M={ }^{t} V \Lambda V$ be the eigen-decomposition of $M$ as in Proposition 2.2. We observe that $\langle x, M x\rangle=\left\langle x,{ }^{t} V \Lambda V x\right\rangle=\langle V x, \Lambda(V x)\rangle$, and since $V$ is orthogonal, $\|V x\|=\|x\|$. Thus, it suffices to consider the case when $M=\Lambda$ is a diagonal matrix with the eigenvalues $\lambda_{1}, \ldots, \lambda_{n}$ in the diagonal. Then we can write

$$
\langle x, M x\rangle=(x(1), \ldots, x(n))\left(\begin{array}{ccc}
\lambda_{1} & & \\
& \ddots & \\
& & \lambda_{n}
\end{array}\right)\left(\begin{array}{c}
x(1) \\
\vdots \\
x(n)
\end{array}\right)=\sum_{i=1}^{n} \lambda_{i} x(i)^{2} .
$$

We note that when $M$ is diagonal, the eigenvectors of $M$ are $v_{k}=e_{k}$, the standard basis vector in $\mathbb{R}^{n}$, i.e., $e_{k}(i)=1$ if $i=k$, and $e_{k}(i)=0$ otherwise. Then the condition $x \in S_{k-1}^{\perp}$ implies $x \perp e_{i}$ for $i=1, \ldots, k-1$, so $x(i)=\left\langle x, e_{i}\right\rangle=0$. Therefore, for $x \in S_{k-1}^{\perp}$ with $\|x\|=1$, we have

$$
\langle x, M x\rangle=\sum_{i=1}^{n} \lambda_{i} x(i)^{2}=\sum_{i=k}^{n} \lambda_{i} x(i)^{2} \leq \lambda_{k} \sum_{i=k}^{n} x(i)^{2}=\lambda_{k}\|x\|^{2}=\lambda_{k} .
$$

On the other hand, letting $x=e_{k} \in S_{k-1}^{\perp}$ yields $\langle x, M x\rangle=\left\langle e_{k}, M e_{k}\right\rangle=\lambda_{k}$. This shows that

$$
\lambda_{k}=\max _{\|x\|=1, x \in S_{k-1}^{\perp}}\langle x, M x\rangle .
$$

Similarly, for $\|x\|=1$,

$$
\langle x, M x\rangle=\sum_{i=1}^{n} \lambda_{i} x(i)^{2} \geq \lambda_{n} \sum_{i=1}^{n} x(i)^{2}=\lambda_{n}\|x\|^{2}=\lambda_{n} .
$$

On the other hand, taking $x=e_{n}$ yields $\langle x, M x\rangle=\left\langle e_{n}, M e_{n}\right\rangle=\lambda_{n}$. Hence we conclude that

$$
\lambda_{n}=\min _{\|x\|=1}\langle x, M x\rangle \text {. }
$$

\subsection{Graphs and matrices}

In this section, we introduce notation and definitions related to algebraic graph theory. Throughout the paper, we consider undirected (positive) weighted regular graphs and we allow self-loops, unless stated otherwise. Here a graph is said to be regular ${ }^{\ddagger}$ if it is $d$-regular for some $d$. Let $G=(V, E, W)$ be a graph, where $V$ is a set of vertices, $|V|=n$,

${ }^{\ddagger}$ When we consider a bipartite graph $G=(U, V, E)$, it is said to be regular if all the vertices in $U$ and $V$ have the same degree $d$ and $d^{\prime}$ respectively for some $d, d^{\prime}$. 
$E \subseteq V \times V$ is a set of edges and $W=\left\{w_{e}\right\}_{e \in E}$ is a set of edge weights. To identify an undirected graph and a matrix later, we think of a graph as a symmetric directed graph, i.e., we assume that if $(u, v) \in E$ and $u \neq v$, then $(v, u) \in E$ holds. The degree of $u \in V$ is defined as

$$
d(u):=\sum_{v \in V:(u, v) \in E} w_{(u, v)}
$$

We say that a graph is $d$-regular if all the vertices have the same degree $d$.

Definition 2.5. For a graph $G$, the adjacency matrix $A=A_{G} \in \mathbb{R}^{V \times V}$ is given by

$$
A(u, v)= \begin{cases}w_{(u, v)} & \text { if }(u, v) \in E, \\ 0 & \text { otherwise. }\end{cases}
$$

If $G$ is $d$-regular, the random walk matrix $\mathcal{A}=\mathcal{A}_{G} \in \mathbb{R}^{V \times V}$ is given by

$$
\mathcal{A}(u, v)= \begin{cases}w_{(u, v)} / d & \text { if }(u, v) \in E, \\ 0 & \text { otherwise. }\end{cases}
$$

Note that $A_{G}$ and $\mathcal{A}_{G}$ are symmetric.

In the rest of this section, we assume that a graph $G$ is $d$-regular. For two sets $S, T \subseteq V$, let

$$
E(S, T):=\sum_{(u, v) \in S \times T:(u, v) \in E} w_{(u, v)}=\sum_{(u, v) \in S \times T} A(u, v) .
$$

Here $(u, v)$ refers to an ordered pair. Thus, we count edges $(u, v), u \neq v$ entirely within $S \cap T$ twice, corresponding to both orientations. We define $\mathcal{E}(S, T)$ as the fraction of edges going from $S$ to $T$, which can be calculated, in the case of $d$-regular graph $G$, as

$$
\mathcal{E}(S, T):=\frac{E(S, T)}{\sum_{(i, j) \in E} w_{(i, j)}}=\frac{E(S, T)}{d \cdot n}=\frac{\sum_{(i, j) \in S \times T} \mathcal{A}(i, j)}{n} .
$$

For a vertex set $S \subseteq V$, we define its (edge) boundary as the fraction of edges leaving $S$ (going from $S$ to the complement of $S)$, i.e., $\mathcal{E}(S, V \backslash S)$. We define its volume $\mu_{G}(S)$ as the fraction of edges going out a vertex in $S$,

$$
\mu_{G}(S):=\varepsilon(S, V) \text {. }
$$

The expansion $\Phi_{G}(S)$ is the ratio of these quantities

$$
\Phi_{G}(S):=\frac{\mathcal{E}(S, V \backslash S)}{\mu_{G}(S)} .
$$

The expansion of the graph $G$ is the minimum expansion of a set with volume at most $1 / 2$,

$$
\Phi_{G}:=\min _{S \subseteq V, \mu_{G}(S) \leq 1 / 2} \Phi_{G}(S) .
$$

We sometimes omit the subscript $G$ if it is clear from the context.

For a set $S \subseteq V$, let $\chi_{S} \in \mathbb{R}^{V}$ denote the characteristic vector of $S$,

$$
\chi_{S}(u)= \begin{cases}1 & \text { if } u \in S \\ 0 & \text { otherwise. }\end{cases}
$$

We redefine the inner product of $x, y \in \mathbb{R}^{V}$ as $\langle x, y\rangle:=\frac{1}{|V|} \sum_{u \in V} x(u) y(u)$ and the norm of $x$ as $\|x\|:=\langle x, x\rangle^{1 / 2}$; then the following identities hold for all vertex sets $S, T \subseteq V$,

$$
\begin{array}{r}
\mathcal{E}(S, T)=\left\langle\chi_{S}, \mathcal{A}_{G} \chi_{T}\right\rangle, \\
\mu(S)=\left\|\chi_{S}\right\|^{2}=|S| /|V|, \\
\mathcal{E}(S, V \backslash S)=\left\langle\chi_{S},\left(I_{V}-\mathcal{A}_{G}\right) \chi_{S}\right\rangle .
\end{array}
$$

Here $I_{V}$ is the $|V| \times|V|$ identity matrix. Note that we assume $G$ is $d$-regular.

\subsection{Cheeger's inequality}

Let $G$ be a $d$-regular graph, $\mathcal{A}_{G}$ be its random walk matrix, and $\lambda_{1} \geq \lambda_{2} \geq \cdots \geq \lambda_{n}$ are the eigenvalues of $\mathcal{A}_{G}$. It is easy to see that $\lambda_{1}=1$ and $v_{1}=(1,1, \ldots, 1)$. We can show that $\lambda_{2}=1$ if and only if $G$ is not connected. We can also show that $\lambda_{n} \geq-1$ and $\lambda_{n}=-1$ if and only if at least one of the connected components of $G$ is bipartite. The spectral gap of $G$, denoted by $\gamma$, is defined as $\gamma=\lambda_{1}-\lambda=1-\lambda$, where $\lambda=\max \left\{\left|\lambda_{2}\right|,\left|\lambda_{n}\right|\right\}$. $\gamma$ quantitatively characterizes the "connectedness" of $G$ in the following sense.

Lemma 2.6 (Cheeger's inequality). Let $G$ be a regular graph and let $1=\lambda_{1} \geq \lambda_{2} \geq \cdots \geq \lambda_{n} \geq-1$ be the eigenvalues of $\mathcal{A}_{G}$. Then, 


$$
\gamma / 2 \leq \Phi_{G} \leq \sqrt{2 \gamma} .
$$

The second inequality of the above lemma follows from the next lemma. The first inequality is the special case of the expander mixing lemma, which is proven in the next section.

Lemma 2.7 (Lemma 2.2 of [48]). For every vector $f \in \mathbb{R}^{V}$, there exists a level set $S \subseteq V$ of the function $f^{2}$ with expansion

$$
\Phi(S) \leq \sqrt{1-\left\langle f, \mathcal{A}_{G} f\right\rangle^{2} /\|f\|^{4}} .
$$

Here "a level set $S \subseteq V$ of the function $f^{2}$ " means that $S$ can be defined as

$$
S:=\left\{u \in V \mid f(u)^{2}>t\right\}
$$

for some $t \in \mathbb{R}$.

Proof. Let $f \in \mathbb{R}^{V}$. Suppose $\max _{u \in V}|f(u)| \leq 1$. Consider the following distribution over vertex subsets $S \subseteq V$ :

(1) Sample $t \in[0,1]$ uniformly at random.

(2) Output the set $S=\left\{u \in V \mid f(u)^{2}>t\right\}$.

Note that every set $S$ in the support of this distribution is a level set of the function $f^{2}$. In the following claims, we establish simple properties of this distribution.

Claim 2.8. The expected volume of $S$ satisfies $\mathbf{E}_{S} \mu(S)=\sum_{T \subseteq V} \mathbf{P}_{S}\{S=T\} \cdot \mu(T)=\|f\|^{2}$.

Proof. We calculate the expected volume as follows.

$$
\underset{S}{\mathbf{E}} \mu(S)=\underset{u \sim V}{\mathbf{E}} \underset{t \in[0,1]}{\mathbf{P}}\left\{f(u)^{2}>t\right\}=\|f\|^{2} .
$$

Here $u \sim V$ means the expectation is with respect to the uniform distribution over $V$.

Claim 2.9. The expected boundary of $S$ is bounded by $\mathbf{E}_{S} \mathcal{E}(S, V \backslash S) \leq\|f\|^{2} \sqrt{1-\left\langle f, \mathcal{A}_{G} f\right\rangle^{2} /\|f\|^{4}}$.

Proof. We calculate the expected boundary of $S$ and apply the Cauchy-Schwarz inequality. From the definition of $\varepsilon(S, T)$, we have

$$
\begin{aligned}
\underset{S}{\mathbf{E}} \mathcal{E}(S, V \backslash S)= & \frac{1}{n} \sum_{(u, v) \in E} \mathcal{A}(u, v) \underset{S}{\mathbf{P}}\{u \in S \wedge v \notin S\}=\frac{1}{n} \sum_{(u, v) \in E} \mathcal{A}(u, v) \underset{t \in[0,1]}{\mathbf{P}}\left\{f(u)^{2}>t \geq f(v)^{2}\right\} \\
= & \frac{1}{n} \sum_{(u, v) \in E} \mathcal{A}(u, v) \max \left\{f(u)^{2}-f(v)^{2}, 0\right\} \\
= & \frac{1}{2} \frac{1}{n} \sum_{(u, v) \in E} \mathcal{A}(u, v)\left|f(u)^{2}-f(v)^{2}\right|=\frac{1}{2} \frac{1}{n} \sum_{(u, v) \in E} \mathcal{A}(u, v)|f(u)-f(v)| \cdot|f(u)+f(v)| \\
\leq & \left(\frac{1}{2} \frac{1}{n} \sum_{(u, v) \in E} \mathcal{A}(u, v)(f(u)-f(v))^{2} \cdot \frac{1}{2} \frac{1}{n} \sum_{(u, v) \in E} \mathcal{A}(u, v)(f(u)+f(v))^{2}\right)^{1 / 2} \\
& (\text { using Cauchy-Schwartz) } \\
= & \left\langle f,\left(I_{V}-\mathcal{A}_{G}\right) f\right\rangle^{1 / 2}\left\langle f,\left(I_{V}+\mathcal{A}_{G}\right) f\right\rangle^{1 / 2}=\sqrt{\|f\|^{4}-\left\langle f, \mathcal{A}_{G} f\right\rangle^{2}} \\
& \left(\text { by } \sum_{u \in V} \mathcal{A}(u, v)=\sum_{v \in V} \mathcal{A}(u, v)=1\right) .
\end{aligned}
$$

We combine the previous claims to complete the proof of Lemma 2.7. Let $S^{*}$ be the level set of $f^{2}$ with minimum expansion. Then,

$$
\begin{aligned}
\Phi\left(S^{*}\right) & \leq \frac{\mathbf{E}_{S} \mathcal{E}(S, V \backslash S)}{\mathbf{E}_{S} \mu(S)}\left(\text { using an inequality } \frac{a}{b} \leq \frac{a+a^{\prime}}{b+b^{\prime}} \text { for } \frac{a}{b} \leq \frac{a^{\prime}}{b^{\prime}}\right) \\
& \leq \frac{\|f\|^{2} \sqrt{1-\left\langle f, \mathcal{A}_{G} f\right\rangle^{2} /\|f\|^{4}}}{\|f\|^{2}} \text { (using Claim 2.8 and Claim 2.9). }
\end{aligned}
$$

Therefore, the set $S^{*}$ satisfies the bound of Lemma 2.7.

\subsection{Expander mixing lemma}

In this section, we prove the following lemma.

Lemma 2.10 (Expander mixing lemma (Lemma 4.15 in [50])). Let $G$ be a d-regular graph and $\mathcal{A}_{G}$ be its random walk matrix with spectral gap $\gamma=1-\lambda$. Then for all sets of vertices $S, T$ of volume $\alpha=\mu(S)$ and $\beta=\mu(T)$, we have

$$
|\mathcal{E}(S, T)-\alpha \beta| \leq \lambda \sqrt{\alpha \cdot(1-\alpha) \cdot \beta \cdot(1-\beta)} .
$$


Proof. Let $\chi_{S}$ be the characteristic (row) vector of $S$ and $\chi_{T}$ the characteristic vector of $T$. Note that $\mathcal{E}(S, T)=\left\langle\chi_{S}, \mathcal{A}_{G} \chi_{T}\right\rangle$.

We can express $\chi_{S}$ as a linear combination of two vectors, one parallel to $\chi_{V}$, and the other a vector $\chi_{S}^{\perp}$, where $\chi_{S}^{\perp} \perp \chi_{V}$. The coefficient of $\chi_{V}$ is $\left\langle\chi_{S}, \chi_{V}\right\rangle=\frac{1}{n} \sum_{i} \chi_{S}(i)=\mu(S)=\alpha$. Then $\chi_{S}=\alpha \chi_{V}+\chi_{S}^{\perp}$ and similarly $\chi_{T}=\beta \chi_{V}+\chi_{T}^{\perp}$.

We have

$$
\begin{aligned}
\mathcal{E}(S, T) & =\left\langle\alpha \chi_{V}+\chi_{S}^{\perp}, \mathcal{A}_{G}\left(\beta \chi_{V}+\chi_{T}^{\perp}\right)\right\rangle \\
& =\alpha \beta\left\langle\chi_{V}, \mathcal{A}_{G} \chi_{V}\right\rangle+\alpha\left\langle\chi_{V}, \mathcal{A}_{G} \chi_{T}^{\perp}\right\rangle+\beta\left\langle\chi_{S}^{\perp}, \mathcal{A}_{G} \chi_{V}\right\rangle+\left\langle\chi_{S}^{\perp}, \mathcal{A}_{G} \chi_{T}^{\perp}\right\rangle .
\end{aligned}
$$

Since ${ }^{t} \chi_{V} \mathcal{A}_{G}={ }^{t} \chi_{V}$ and $\mathscr{A}_{G} \chi_{V}=\chi_{V}$, and both $\chi_{S}^{\perp}$ and $\chi_{T}^{\perp}$ are orthogonal to $\chi_{V}$, the above expression is simplified:

$$
\mathcal{E}(S, T)=\alpha \beta\left\langle\chi_{V}, \chi_{V}\right\rangle+\left\langle\chi_{S}^{\perp}, \mathcal{A}_{G} \chi_{T}^{\perp}\right\rangle=\alpha \beta+\left\langle\chi_{S}^{\perp}, \mathcal{A}_{G} \chi_{T}^{\perp}\right\rangle .
$$

Thus,

$$
\begin{aligned}
|\mathcal{E}(S, T)-\alpha \beta| & =\left|\left\langle\chi_{S}^{\perp}, \mathcal{A}_{G} \chi_{T}^{\perp}\right\rangle\right| \\
& \leq\left\|\chi_{S}^{\perp}\right\| \cdot\left\|\mathcal{A}_{G} \chi_{T}^{\perp}\right\| \\
& \leq \lambda\left\|\chi_{S}^{\perp}\right\| \cdot\left\|\chi_{T}^{\perp}\right\|,
\end{aligned}
$$

where the last inequality is obtained by regarding $\chi_{T}^{\perp}$ as a linear combination of eigenvectors of $\mathcal{A}_{G}$ and recalling the definition of spectral gap. To complete the proof, we note that

$$
\alpha=\left\|\chi_{S}\right\|^{2}=\left\|\alpha \chi_{V}\right\|^{2}+\left\|\chi_{S}^{\perp}\right\|^{2}=\alpha^{2}+\left\|\chi_{S}^{\perp}\right\|^{2},
$$

so $\left\|\chi_{S}^{\perp}\right\|=\sqrt{\left(\alpha-\alpha^{2}\right)}=\sqrt{\alpha \cdot(1-\alpha)}$ and similarly $\left\|\chi_{T}^{\perp}\right\|=\sqrt{\left(\beta-\beta^{2}\right)}=\sqrt{\beta \cdot(1-\beta)}$.

\section{Analytical Proof of Parallel Repetition Theorem}

In this section, we describe the proof of the parallel repetition theorem for a special case of regular projection games due to [17] basically following the presentation of Version v1 of [16].

In what follows, we consider a regular projection game $G=(U, V, E, \Sigma, W, \Pi)$ and identify it with the following matrix in $\mathbb{R}^{(U \times \Sigma) \times(V \times \Sigma)}$,

$$
G((u, \alpha),(v, \beta))= \begin{cases}w_{(u, v)} / d & \text { if }(u, v) \in E \text { and }(\alpha, \beta) \in \pi_{(u, v)}, \\ 0 & \text { otherwise. }\end{cases}
$$

Here, a game is said to be regular if there exist two real numbers $d$ and $d^{\prime}$ such that the vertices $u \in U$ have degree $d$, i.e., $d(u)=\sum_{v:(u, v) \in E} w_{(u, v)}=d$ for any $u \in U$ and the vertices in $V$ have degree $d^{\prime}$.

We identify an assignment for the vertex set $U$ with a 0-1 vector $f \in \mathbb{R}^{U \times \Sigma}$ such that $f(u, \gamma)=1$ if $u \in U$ is assigned the label $\gamma \in \Sigma$ and $f(u, \gamma)=0$ otherwise. Similarly, we identify an assignment for the vertex set $V$ with a 0 -1 vector $g \in \mathbb{R}^{V \times \Sigma}$. A vector $f \in\{0,1\}^{U \times \Sigma}$ (resp., $g \in\{0,1\}^{V \times \Sigma}$ ) is called a partial assignment if $\sum_{\alpha \in \Sigma} f(u, \alpha) \leq 1$ (resp., $\sum_{\beta \in \Sigma} g(v, \beta) \leq 1$ ) holds for any $u \in U$ (resp., any $v \in V$ ).

We define the inner product of $x, y \in \mathbb{R}^{U \times \Sigma}$ and the norm of $x \in \mathbb{R}^{U \times \Sigma}$ with respect to $U$ and $\Sigma$ as

$$
\langle x, y\rangle_{U}:=\frac{1}{|U|} \sum_{u \in U, \alpha \in \Sigma} x(u, \alpha) y(u, \alpha),\|x\|_{U}:=\sqrt{\langle x, x\rangle_{U}} .
$$

The following observation is useful.

Claim 3.1. Given two assignments $f \in\{0,1\}^{U \times \Sigma}$ and $g \in\{0,1\}^{V \times \Sigma}$, the normalized sum of the weights of satisfied edges is exactly equal to $\langle f, G g\rangle_{U}$. Specifically, $\operatorname{val}(G)=\max _{f, g}\langle f, G g\rangle_{U}$ holds.

Proof. By definition, we have

$$
(G g)(u, \alpha)=\frac{1}{d} \sum_{v \in V:(u, v) \in E} w_{(u, v)} \sum_{\beta:(\alpha, \beta) \in \pi_{(u, v)}} g(v, \beta) .
$$

Let $f^{\prime}: U \rightarrow \Sigma$ and $g^{\prime}: V \rightarrow \Sigma$ be assignments corresponding to $f$ and $g$. Then,

$$
\begin{aligned}
\langle f, G g\rangle_{U} & =\frac{1}{|U|} \sum_{u \in U, \alpha \in \Sigma} f(u, \alpha) \times \frac{1}{d} \sum_{v \in V:(u, v) \in E} w_{(u, v)} \sum_{\beta:(\alpha, \beta) \in \pi_{(u, v)}} g(v, \beta) \\
& =\frac{1}{d|U|} \sum_{(u, v) \in E,(\alpha, \beta) \in \pi_{(u, v)}} w_{(u, v)} f(u, \alpha) g(v, \beta) \\
& =\frac{1}{\sum_{e \in E} w_{e}} \sum_{(u, v) \in E:\left(f^{\prime}(u), g^{\prime}(v)\right) \in \pi_{(u, v)}} w_{(u, v)} .
\end{aligned}
$$


We remark that this setup gives a description of the value of the game as $\max _{f, g}\langle f, G g\rangle_{U}$, which is quite similar to the largest eigenvalue of the matrix characterized in Theorem 2.4, except that the maximum is taken only over $0-1$ vectors such that $\sum_{\gamma} f(u, \gamma)=1$ for all $u \in U$ and $\sum_{\gamma} g(v, \gamma)=1$ for all $v \in V$.

Given an assignment $g \in\{0,1\}^{V \times \Sigma}$, the collision value of $G$ with respect to $g$, defined as the norm $\|G g\|_{U}$, is a reasonable measure of the maximal value of the game using $g$ in the following sense.

Claim 3.2. Let $G \in \mathbb{R}^{(U \times \Sigma) \times(V \times \Sigma)}$ be a regular projection game. Then $\operatorname{val}(G)^{2} \leq \max _{g}\|G g\|_{U}^{2} \leq \operatorname{val}(G)$, where the maximum is taken over all assignments $g \in\{0,1\}^{V \times \Sigma}$.

Proof. For the second inequality, let $g \in\{0,1\}^{V \times \Sigma}$ be an assignment for $G$. Note that $\sum_{\alpha \in \Sigma}(G g)(u, \alpha)=1$ holds for any $u \in U$. This is because $\sum_{(\alpha, \beta) \in \pi_{(u, v)}} g(v, \beta)=1$ for any $(u, v) \in E$ as $g$ is an assignment and $\pi(u, v)$ is a projection constraint. Then,

$$
\|G g\|_{U}^{2}=\langle G g, G g\rangle_{U} \leq\langle\widetilde{f}, G g\rangle_{U} \leq \max _{f^{\prime}, g^{\prime}}\left\langle f^{\prime}, G g^{\prime}\right\rangle_{U}=\operatorname{val}(G)
$$

where the last equality is by Claim 3.1 and we define an assignment $\widetilde{f} \in\{0,1\}^{U \times \Sigma}$ as

$$
\widetilde{f}(u, \alpha)= \begin{cases}1 & \text { if } \alpha=\arg \max _{\gamma \in \Sigma}\{(G g)(u, \gamma)\}, \\ 0 & \text { otherwise. }\end{cases}
$$

In the definition of $\tilde{f}$, we break ties of arg max arbitrarily but in some fixed manner.

If $f, g$ are optimal assignments, then it holds that

$$
\operatorname{val}(G)^{2}=\langle f, G g\rangle_{U}^{2} \leq\|f\|_{U}^{2} \cdot\|G g\|_{U}^{2}=\|G g\|_{U}^{2} .
$$

Given two games $G \in \mathbb{R}^{(U \times \Sigma) \times(V \times \Sigma)}$ and $G^{\prime} \in \mathbb{R}^{\left(U^{\prime} \times \Sigma^{\prime}\right) \times\left(V^{\prime} \times \Sigma^{\prime}\right)}$, define the product game $G \otimes G^{\prime} \in$ $\mathbb{R}^{\left(U \times U^{\prime} \times \Sigma \times \Sigma^{\prime}\right) \times\left(V \times V^{\prime} \times \Sigma \times \Sigma^{\prime}\right)}$ by the tensor product of the respective matrices. More explicitly,

$$
\left(G \otimes G^{\prime}\right)\left(u, u^{\prime}, \alpha, \alpha^{\prime}, v, v^{\prime}, \beta, \beta^{\prime}\right)=G(u, \alpha, v, \beta) \cdot G^{\prime}\left(u^{\prime}, \alpha^{\prime}, v^{\prime}, \beta^{\prime}\right) .
$$

Note that if we regard $G, G^{\prime}$ as games in the original sense and define $G \otimes G^{\prime}$ as in Definition 1.2, then the matrix corresponding to the game $G \otimes G^{\prime}$ is as the same as the matrix given above.

We define $G^{\otimes k}=G \otimes G^{\otimes k-1}$ inductively in the obvious way. Note that $G^{\otimes k} \in \mathbb{R}^{\left(U^{k} \times \Sigma^{k}\right) \times\left(V^{k} \times \Sigma^{k}\right)}$ and assignments for $G^{\otimes k}$ are $f \in\{0,1\}^{U^{k} \times \Sigma^{k}}$ and $g \in\{0,1\}^{V^{k} \times \Sigma^{k}}$.

Recall that a graph has a spectral gap $\gamma$ if its random walk matrix has eigenvalues $1=\lambda_{1} \geq \lambda_{2} \geq \cdots$ such that $\gamma=\lambda_{1}-\lambda_{2}$. We denote by ${ }^{t} G G$ the game obtained by multiplying two matrices ${ }^{t} G$ and $G$. Our goal is to establish the following parallel repetition theorem:

Theorem 3.3 (Theorem 6 of [17]). Suppose the constraint graph of ${ }^{t} G G$ has a spectral gap $\gamma$ for some $\gamma>0$. If $\operatorname{val}\left(G^{\otimes k}\right) \geq(1-\eta)^{k}$, then $\operatorname{val}(G) \geq 1-O(\eta / \gamma)$.

Let us assume $\operatorname{val}\left(G^{\otimes k}\right) \geq(1-\eta)^{k}$. This implies that there is an assignment $g$ such that $\left\|G^{\otimes k} g\right\|_{U^{k}} \geq(1-\eta)^{k}$ (by Claim 3.2). Our goal is to deduce from $g$ an assignment $\varphi \in\{0,1\}^{V \times \Sigma}$ for $G$ such that $\|G \varphi\|_{U} \geq 1-\bar{O}(\eta / \gamma)$, which immediately implies that $\operatorname{val}(G) \geq 1-O(\eta / \gamma)$ by Claim 3.2.

The proof for the existence of such an assignment $\varphi$ consists of two lemmas. The first lemma, which is the main ingredient of the proof, shows that a good assignment $g \in\{0,1\}^{V^{k} \times \Sigma^{k}}$ for $G^{\otimes k}$ implies a good fractional assignment $h^{\star} \in\{0,1\}^{V \times \Sigma}$ for $G$. A vector $g \in \mathbb{R}^{V \times \Sigma}$ is a fractional assignment if for every vertex $v \in V$, there exists at most one label $\beta \in \Sigma$ with $g(v, \beta) \neq 0$. (In other words, the support of $g$ corresponds to a partial assignment.)

Lemma 3.4 (Lemma 3.1 in Version v1 of [16]). If there exists an assignment $g \in\{0,1\}^{V^{k} \times \Sigma^{k}}$ such that $\left\|G^{\otimes k} g\right\|_{U^{k}} \geq$ $(1-\eta)^{k}$, then there exists a fractional assignment $h^{\star} \in \mathbb{R}^{V \times \Sigma}$ such that $\left\|G h^{\star}\right\|_{U} \geq(1-\eta)\left\|h^{\star}\right\|_{V}$.

The second lemma shows that fractional assignments can be "rounded" to assignments in a way that they approximately preserve the relation between the norms. Furthermore, if the constraint graph of ${ }^{t} G G$ has a spectral gap $\gamma>0$, then the rounded assignments give roughly the same collision values as the norms.

Lemma 3.5 (Lemma 3.2 in Version v1 of [16]). If there exists a fractional assignment $g \in \mathbb{R}^{V \times \Sigma}$ for $G$ with $\|G g\|_{U} \geq(1-\eta)\|g\|_{V}$, and the constraint graph of ${ }^{t} G G$ has a spectral gap $\gamma$ for some $\gamma>0$, then there exists an assignment $\varphi \in\{0,1\}^{V \times \Sigma}$ for $G$ such that $\|G \varphi\|_{U} \geq 1-O(\eta / \gamma)$.

Proof of Lemma 3.4. We will prove Lemma 3.4 by induction on $k$. Suppose $\left\|G^{\otimes k} g\right\|_{U^{k}} \geq(1-\eta)^{k}$. If $k=1$, we are done by setting $h^{\star}=g$ since $\left\|h^{\star}\right\|_{V}=\|g\|_{V}=1$. Suppose $k>1$. Then, we can assume

$$
\max _{g^{\prime}}\left\|G^{\otimes(k-1)} g^{\prime}\right\|_{U^{k-1}} \leq(1-\eta)^{k-1} .
$$

(Otherwise, we would be done by induction hypothesis.)

We can write $G^{\otimes k}=G \otimes H$ for $H=G^{\otimes(k-1)}$. The game $H=G^{\otimes(k-1)}$ is the one with vertex sets $U_{H}=U^{k-1}$ and $V_{H}=V^{k-1}$, and alphabet $\Sigma_{H}=\Sigma^{k-1}$.

Let $F=\left(V, V, E_{F}, \Sigma,\left\{w_{e}\right\}, \Pi_{F}\right)$ be the following trivial game corresponding to $G: V, \Sigma$ are the same sets as those of $G$ and we assume $\Sigma=\{1,2, \ldots,|\Sigma|\}$. The edge set is $E_{F}=\{(v, v) \in V \times V \mid v \in V\}, w_{e}=1$ and $\pi_{e}=\{(1, \beta) \mid \beta \in \Sigma\}$ 
for each $e \in E_{F}$. Namely, $F\left(\left(v_{1}, \alpha\right),\left(v_{2}, \beta\right)\right)=1$ if $v_{1}=v_{2}$ and $\alpha=1$ and 0 otherwise. By definition, we see that $\|F g\|_{V}=1$ for all assignments $g$ for $V$.

Claim 3.6. Let $F$ be the trivial game and $G^{\prime} \in \mathbb{R}^{\left(U^{\prime} \times \Sigma^{\prime}\right) \times\left(V^{\prime} \times \Sigma^{\prime}\right)}$ be a game. Then, for any assignment $g \in\{0,1\}^{V \times V^{\prime} \times \Sigma \times \Sigma^{\prime}}$ for $F \otimes G^{\prime}$, there exists an assignment $g^{\prime} \in\{0,1\}^{V^{\prime} \times \Sigma^{\prime}}$ for $G^{\prime}$ such that

holds.

$$
\left\|\left(F \otimes G^{\prime}\right) g\right\|_{V \times U^{\prime}} \leq\left\|G^{\prime} g^{\prime}\right\|_{U^{\prime}}
$$

Proof. First note that $\left(F \otimes G^{\prime}\right) g \in \mathbb{R}^{V \times U^{\prime} \times \Sigma \times \Sigma^{\prime}}$. We see that $\left(\left(F \otimes G^{\prime}\right) g\right)\left(v, u^{\prime}, \alpha, \alpha^{\prime}\right)=0$ for any $v \in V, u^{\prime} \in U^{\prime}, \alpha^{\prime} \in \Sigma^{\prime}$ and $\alpha \neq 1$. Thus, we have

$$
\begin{aligned}
\left\|\left(F \otimes G^{\prime}\right) g\right\|_{V \times U^{\prime}}^{2} & =\frac{1}{|V|\left|U^{\prime}\right|} \sum_{v \in V, u^{\prime} \in U^{\prime}, \alpha^{\prime} \in \Sigma^{\prime}}\left(\left(F \otimes G^{\prime}\right) g\right)\left(v, u^{\prime}, 1, \alpha^{\prime}\right)^{2} \\
& =\frac{1}{|V|} \sum_{v \in V} \frac{1}{\left|U^{\prime}\right|} \sum_{u^{\prime} \in U^{\prime}, \alpha^{\prime} \in \Sigma^{\prime}}\left(\left(F \otimes G^{\prime}\right) g\right)\left(v, u^{\prime}, 1, \alpha^{\prime}\right)^{2} \\
& \leq \max _{v \in V} \frac{1}{\left|U^{\prime}\right|} \sum_{u^{\prime} \in U^{\prime}, \alpha^{\prime} \in \Sigma^{\prime}}\left(\left(F \otimes G^{\prime}\right) g\right)\left(v, u^{\prime}, 1, \alpha^{\prime}\right)^{2} .
\end{aligned}
$$

We denote by $v^{\star} \in V$ a vertex achieving the maximum in the above expression. Now we define $g^{\prime} \in\{0,1\}^{V^{\prime} \times \Sigma^{\prime}}$ as

$$
g^{\prime}\left(v^{\prime}, \beta^{\prime}\right)=\sum_{\beta \in \Sigma} g\left(v^{\star}, v^{\prime}, \beta, \beta^{\prime}\right) .
$$

By the choice of $v^{\star}$, we have

$$
\begin{aligned}
\left\|G^{\prime} g^{\prime}\right\|_{U^{\prime}}^{2} & =\frac{1}{\left|U^{\prime}\right|} \sum_{u^{\prime} \in U^{\prime}, \alpha^{\prime} \in \Sigma^{\prime}}\left(G^{\prime} g^{\prime}\right)\left(u^{\prime}, \alpha^{\prime}\right)^{2} \\
& =\frac{1}{\left|U^{\prime}\right|} \sum_{u^{\prime} \in U^{\prime}, \alpha^{\prime} \in \Sigma^{\prime}}\left(\left(F \otimes G^{\prime}\right) g\right)\left(v^{\star}, u^{\prime}, 1, \alpha^{\prime}\right)^{2} \geq\left\|\left(F \otimes G^{\prime}\right) g\right\|_{V \times U^{\prime}}^{2},
\end{aligned}
$$

where the second equality is by

$$
\begin{aligned}
\left(\left(F \otimes G^{\prime}\right) g\right)\left(v^{\star}, u^{\prime}, 1, \alpha^{\prime}\right) & =\sum_{v, v^{\prime}, \beta, \beta^{\prime}} F\left(v^{\star}, 1, v, \beta\right) \cdot G\left(u^{\prime}, \alpha^{\prime}, v^{\prime}, \beta^{\prime}\right) \cdot g\left(v, v^{\prime}, \beta, \beta^{\prime}\right) \\
& =\sum_{v^{\prime}, \beta^{\prime}} G^{\prime}\left(u^{\prime}, \alpha^{\prime}, v^{\prime}, \beta^{\prime}\right) \sum_{\beta} g\left(v^{\star}, v^{\prime}, \beta, \beta^{\prime}\right) \\
& =\sum_{v^{\prime}, \beta^{\prime}} G^{\prime}\left(u^{\prime}, \alpha^{\prime}, v^{\prime}, \beta^{\prime}\right) \cdot g^{\prime}\left(v^{\prime}, \beta^{\prime}\right)=\left(G^{\prime} g^{\prime}\right)\left(u^{\prime}, \alpha^{\prime}\right) .
\end{aligned}
$$

This completes the proof.

Since

$$
\|(F \otimes H) g\|_{V \times U^{k-1}} \leq \max _{g^{\prime}}\left\|(F \otimes H) g^{\prime}\right\|_{V \times U^{k-1}} \leq \max _{g^{\prime \prime}}\left\|H g^{\prime \prime}\right\|_{U^{k-1}} \leq(1-\eta)^{k-1}
$$

(using Claim 3.6 and (3.1)), we get

$$
\|(G \otimes H) g\|_{U^{k}} \geq(1-\eta)^{k} \geq(1-\eta) \cdot\|(F \otimes H) g\|_{V \times U^{k-1}}
$$

and we will argue that this allows us to extract a fractional assignment for $G$ with value at least $1-\eta$.

Claim 3.7. We have $G \otimes H=\left(G \otimes I_{U_{H} \times \Sigma_{H}}\right)\left(I_{V \times \Sigma} \otimes H\right)$ and $F \otimes H=\left(F \otimes I_{U_{H} \times \Sigma_{H}}\right)\left(I_{V \times \Sigma} \otimes H\right)$.

Proof. We only prove the first equality. The second equality can be proved similarly. First note that $G \otimes H \in$ $\mathbb{R}^{\left(U \times U_{H} \times \Sigma \times \Sigma_{H}\right) \times\left(V \times V_{H} \times \Sigma \times \Sigma_{H}\right)}, \quad G \otimes I_{U_{H} \times \Sigma_{H}} \in \mathbb{R}^{\left(U \times U_{H} \times \Sigma \times \Sigma_{H}\right) \times\left(V \times U_{H} \times \Sigma \times \Sigma_{H}\right)}, \quad I_{V \times \Sigma} \otimes H \in \mathbb{R}^{\left(V \times U_{H} \times \Sigma \times \Sigma_{H}\right) \times\left(V \times V_{H} \times \Sigma \times \Sigma_{H}\right)}$, thus, the above matrix multiplication is well-defined. By definition, we have

$$
\left(G \otimes I_{U_{H} \times \Sigma_{H}}\right)\left(u, u_{H}, \alpha, \alpha_{H}, v, u_{H}^{\prime}, \beta, \beta_{H}\right)= \begin{cases}G(u, \alpha, v, \beta) & \text { if } u_{H}=u_{H}^{\prime}, \alpha_{H}=\beta_{H}, \\ 0 & \text { otherwise }\end{cases}
$$

and

$$
\left(I_{V \times \Sigma} \otimes H\right)\left(v, u_{H}, \alpha, \alpha_{H}, v^{\prime}, v_{H}, \beta, \beta_{H}\right)= \begin{cases}H\left(u_{H}, \alpha_{H}, v_{H}, \beta_{H}\right) & \text { if } v=v^{\prime}, \alpha=\beta, \\ 0 & \text { otherwise. }\end{cases}
$$

We can calculate as 
$\left(\left(G \otimes I_{U_{H} \times \Sigma_{H}}\right)\left(I_{V \times \Sigma} \otimes H\right)\right)\left(u, u_{H}, \alpha, \alpha_{H}, v, v_{H}, \beta, \beta_{H}\right)$

$$
\begin{aligned}
& =\sum_{v^{\prime}, u_{H}^{\prime}, \beta^{\prime}, \beta_{H}^{\prime}}\left(G \otimes I_{U_{H} \times \Sigma_{H}}\right)\left(u, u_{H}, \alpha, \alpha_{H}, v^{\prime}, u_{H}^{\prime}, \beta^{\prime}, \beta_{H}^{\prime}\right) \times\left(I_{V \times \Sigma} \otimes H\right)\left(v^{\prime}, u_{H}^{\prime}, \beta^{\prime}, \beta_{H}^{\prime}, v, v_{H}, \beta, \beta_{H}\right) \\
& =G(u, \alpha, v, \beta) \cdot H\left(u_{H}, \alpha_{H}, v_{H}, \beta_{H}\right)=(G \otimes H)\left(u, u_{H}, \alpha, \alpha_{H}, v, v_{H}, \beta, \beta_{H}\right) .
\end{aligned}
$$

This matrix product can be interpreted as first mapping $g$ to $h=\left(I_{V \times \Sigma} \otimes H\right) g$, and then mapping $h$ to $\left(G \otimes I_{\Omega}\right) h$, where $\Omega=U_{H} \times \Sigma_{H}$. The vector $h$ is in $\mathbb{R}^{V \times U_{H} \times \Sigma \times \Sigma_{H}}$. Rewriting (3.2) by Claim 3.7, we get

$$
\left\|\left(G \otimes I_{\Omega}\right) h\right\|_{U^{k}} \geq(1-\eta)\left\|\left(F \otimes I_{\Omega}\right) h\right\|_{V \times U^{k-1}} .
$$

Let us regard the vector $h$ as a collection of vectors $\left\{h_{\omega} \in \mathbb{R}^{V \times \Sigma}\right\}_{\omega \in \Omega}$. Then, we get $\left\|\left(G \otimes I_{\Omega}\right) h\right\|_{U^{k}}^{2}=$ $\frac{1}{\left|U_{H}\right|} \sum_{\omega \in \Omega}\left\|G h_{\omega}\right\|_{U}^{2}$ and similarly $\left\|\left(F \otimes I_{\Omega}\right) h\right\|_{V \times U^{k-1}}^{2}=\frac{1}{\left|U_{H}\right|} \sum_{\omega \in \Omega}\left\|F h_{\omega}\right\|_{V}^{2}$. For example, the first equality can be shown as follows:

$$
\begin{aligned}
\left\|\left(G \otimes I_{\Omega}\right) h\right\|_{U^{k}}^{2} & =\frac{1}{|U| \cdot\left|U_{H}\right|} \sum_{u, u_{H}, \alpha, \alpha_{H}}\left(\left(G \otimes I_{\Omega}\right) h\right)\left(u, u_{H}, \alpha, \alpha_{H}\right)^{2} \\
& =\frac{1}{|U| \cdot\left|U_{H}\right|} \sum_{u, u_{H}, \alpha, \alpha_{H}}\left(\sum_{v, u_{H}^{\prime}, \beta, \alpha_{H}^{\prime}} G(u, \alpha, v, \beta) \cdot I_{\Omega}\left(u_{H}, \alpha_{H}, u_{H}^{\prime}, \alpha_{H}^{\prime}\right) \cdot h\left(v, u_{H}^{\prime}, \beta, \alpha_{H}^{\prime}\right)\right)^{2} \\
& =\frac{1}{|U| \cdot\left|U_{H}\right|} \sum_{u, u_{H}, \alpha, \alpha_{H}}\left(\sum_{v, \beta} G(u, \alpha, v, \beta) \cdot h\left(v, u_{H}, \beta, \alpha_{H}\right)\right)^{2} \\
& =\frac{1}{|U| \cdot\left|U_{H}\right|} \sum_{u, u_{H}, \alpha, \alpha_{H}}\left(G h_{\left(u_{H}, \alpha_{H}\right)}\right)(u, \alpha)^{2}=\frac{1}{\left|U_{H}\right|} \sum_{\omega \in \Omega}\left\|G h_{\omega}\right\|_{U}^{2} .
\end{aligned}
$$

There must therefore be some $\omega \in \Omega$ such that $\left\|G h_{\omega}\right\|_{U}^{2} \geq(1-\eta)^{2}\left\|F h_{\omega}\right\|_{V}^{2}$.

It turns out that we can greedily change $h_{\omega}$ to a fractional assignment $h^{\star} \in \mathbb{R}^{V \times \Sigma}$ with $\left\|G h^{\star}\right\|_{U} \geq\left\|G h_{\omega}\right\|_{U}$ and $\left\|h^{\star}\right\|_{V}=\left\|F h_{\omega}\right\|_{V}$. For notational convenience, assume $V=\left\{v_{1}, v_{2}, \ldots, v_{|V|}\right\}$. Define $h_{0}:=h_{\omega}$ and $h_{i}:=$ $\arg \max _{h^{\prime}}\left\langle h^{\prime},{ }^{t} G G h_{\omega}\right\rangle$ where the maximum is over $h^{\prime} \in \mathbb{R}^{V \times \Sigma}$ such that $\sum_{\beta} h^{\prime}\left(v_{i}, \beta\right)=\sum_{\beta} h_{\omega}\left(v_{i}, \beta\right), h^{\prime}\left(v_{i}, \beta\right) \neq 0$ for at most one $\beta \in \Sigma$, and for any $v \in V \backslash\left\{v_{i}\right\}, \beta \in \Sigma, h^{\prime}(v, \beta)=h_{i-1}(v, \beta)$. It is easy to see that $\left\|F h_{i}\right\|=\left\|F h_{i-1}\right\|$. Since $h_{i}\left(v_{i}, \beta\right) \neq 0$ means ${ }^{t} G G h_{\omega}\left(v_{i}, \beta\right) \geq{ }^{t} G G h_{\omega}\left(v_{i}, \beta^{\prime}\right)$ for any $\beta^{\prime} \neq \beta$, we have $\left\langle h_{i},{ }^{t} G G h_{\omega}\right\rangle \geq\left\langle h_{i-1}{ }^{t} G G h_{\omega}\right\rangle$. Thus, by setting $h^{\star}=h_{|V|}$, we have $\left\|G h^{\star}\right\|_{U} \geq(1-\eta)\left\|F h^{\star}\right\|_{V}=(1-\eta)\left\|h^{\star}\right\|_{V}$.

Proof of Lemma 3.5. It suffices to prove $\|G \varphi\|_{U}^{2} \geq 1-O(\eta / \gamma)$ for some assignment $\varphi$ since $\|G \varphi\|_{U} \geq\|G \varphi\|_{U}^{2}$ if $\|G \varphi\|_{U} \leq 1$. Let $g \in \mathbb{R}^{V \times \Sigma}$ be a fractional assignment for $G$ with value $\|G g\|_{U} \geq(1-\eta)\|g\|_{V}$. Without loss of generality, we assume $\|g\|_{V}=1$. Let $x \in \Sigma^{V}$ be an assignment for $V$ that is compatible with $g$ in the sense that $x(v)=\beta$ if $g(v, \beta) \neq 0$. Define an assignment $g^{\prime} \in \mathbb{R}^{V \times \Sigma}$ as $g^{\prime}(v, \beta)=1$ if $x(v)=\beta$ and 0 otherwise.

We consider a probability distribution $\mu$ over $\left(u, v, v^{\prime}\right) \in U \times V^{2}$ corresponding to the following random process: Pick $u \in U$ uniformly at random and pick $v, v^{\prime} \in V$ independently at random with probability induced by edge weights $\left\{w_{(u, v)}\right\}_{(u, v) \in E}$. We define a matrix $Q \in \mathbb{R}^{V \times V}$ from $G$ and $x$ as

$$
Q\left(v, v^{\prime}\right):=\underset{u \mid\left(v, v^{\prime}\right)}{\mathbf{P}}\left[\exists \alpha \in \Sigma,(\alpha, x(v)) \in \pi_{(u, v)} \wedge\left(\alpha, x\left(v^{\prime}\right)\right) \in \pi_{\left(u, v^{\prime}\right)}\right],
$$

where $u \mid\left(v, v^{\prime}\right)$ means that $u$ is sampled under the condition that $v$ and $v^{\prime}$ are picked. Note that the marginal distribution on $V$ is uniform, i.e., $\mathbf{P}_{\left(u, v, v^{\prime}\right)}[v=w]=1 /|V|$ for any $w \in V$ since $G$ is regular. Then, we have

Claim 3.8.

$$
\max _{\varphi}\|G \varphi\|_{U}^{2} \geq\left\|G g^{\prime}\right\|_{U}^{2}=\underset{\left(v, v^{\prime}\right)}{\mathbf{E}}\left[Q\left(v, v^{\prime}\right)\right]
$$

where the expectation is with respect to $\mu$ (but $u$ is omitted in the subscript).

Proof. The inequality is trivial. To establish the equation, we calculate $\mathbf{E}_{\left(v, v^{\prime}\right)}\left[Q\left(v, v^{\prime}\right)\right]$ as follows.

$$
\begin{aligned}
& \underset{\left(v, v^{\prime}\right)}{\mathbf{E}}\left[Q\left(v, v^{\prime}\right)\right]=\underset{\left(u, v, v^{\prime}\right)}{\mathbf{P}}\left[\exists \alpha \in \Sigma,(\alpha, x(v)) \in \pi_{(u, v)} \wedge\left(\alpha, x\left(v^{\prime}\right)\right) \in \pi_{\left(u, v^{\prime}\right)}\right] \\
& =\sum_{a \in U} \underset{\left(u, v, v^{\prime}\right)}{\mathbf{P}}[u=a] \cdot \underset{\left(v, v^{\prime}\right) \mid u=a}{\mathbf{P}}\left[\exists \alpha \in \Sigma,(\alpha, x(v)) \in \pi_{(a, v)} \wedge\left(\alpha, x\left(v^{\prime}\right)\right) \in \pi_{\left(a, v^{\prime}\right)}\right] \\
& =\frac{1}{|U|} \sum_{a \in U} \mathbf{P}_{\left(v, v^{\prime}\right) \mid u=a}\left[\exists \alpha \in \Sigma,(\alpha, x(v)) \in \pi_{(a, v)} \wedge\left(\alpha, x\left(v^{\prime}\right)\right) \in \pi_{\left(a, v^{\prime}\right)}\right] \\
& =\frac{1}{|U|} \sum_{a \in U, \alpha \in \Sigma} \underset{\left(v, v^{\prime}\right) \mid u=a}{\mathbf{P}}\left[(\alpha, x(v)) \in \pi_{(a, v)} \wedge\left(\alpha, x\left(v^{\prime}\right)\right) \in \pi_{\left(a, v^{\prime}\right)}\right]
\end{aligned}
$$




$$
\begin{aligned}
& =\frac{1}{|U|} \sum_{a \in U, \alpha \in \Sigma, b, b^{\prime} \in V} \frac{w_{(a, b)}}{d} \cdot \frac{w_{\left(a, b^{\prime}\right)}}{d} \sum_{\beta:(\alpha, \beta) \in \pi_{(a, b)}} g^{\prime}(b, \beta) \sum_{\beta^{\prime}:\left(\alpha, \beta^{\prime}\right) \in \pi_{\left(a, b^{\prime}\right)}} g^{\prime}\left(b^{\prime}, \beta^{\prime}\right) \\
& =\frac{1}{|U|} \sum_{a \in U, \alpha \in \Sigma}\left(G g^{\prime}\right)(a, \alpha)^{2}=\left\|G g^{\prime}\right\|_{U}^{2} .
\end{aligned}
$$

Here we use the facts $\mathbf{P}_{\left(u, v, v^{\prime}\right)}[u=a]=1 /|U|, v$ and $v^{\prime}$ are independent under $u=a$ and $\mathbf{P}_{v \mid u=a}[v=b]=w_{(a, b)} / d$. The last equality is obtained similarly to the proof of Claim 3.1 .

Let $h \in \mathbb{R}^{V}$ be the vector given by $h(v)=\sum_{\beta} g(v, \beta)$. Since $g$ is a fractional assignment, for each $v \in V$ with $h(v) \neq 0$, there uniquely exists $\beta \in \Sigma$ with $h(v)=g(v, \beta)$. Thus, we have $\|h\|=\|g\|_{V}=1$. We have the following in a similar way to the proof of the above claim.

Claim 3.9.

$$
\underset{\left(v, v^{\prime}\right)}{\mathbf{E}}\left[h(v) h\left(v^{\prime}\right) Q\left(v, v^{\prime}\right)\right]=\|G g\|_{U}^{2} \geq(1-\eta)^{2} .
$$

Let $\hat{G}$ be the constraint graph of ${ }^{t} G G$ and $\mathcal{A}_{\hat{G}} \in \mathbb{R}^{V \times V}$ be its random walk matrix. Since $G$ is regular, so is $\hat{G}$ and we have $\mathcal{A}_{\hat{G}} \chi_{V}=\chi_{V}$ where ${ }^{t} \chi_{V}=(1,1, \ldots, 1)$. Now let us represent $h$ as $h=\left\langle h, \chi_{V}\right\rangle \cdot \chi_{V}+h^{\perp}$. Note that $1=\|h\|^{2}=\left\langle h, \chi_{V}\right\rangle^{2}\left\|\chi_{V}\right\|^{2}+\left\|h^{\perp}\right\|^{2}$. Then, we have

$$
\left\langle h, \mathcal{A}_{\hat{G}} h\right\rangle \leq\left\langle h, \chi_{V}\right\rangle^{2}\left\|\chi_{V}\right\|^{2}+(1-\gamma)\left\|h^{\perp}\right\|^{2}=1-\gamma\left\|h^{\perp}\right\|^{2} .
$$

by calculation similar to the proof of Lemma 2.10 . Combining this bound with $\left\langle h, \mathcal{A}_{\hat{G}} h\right\rangle=\left\langle g,{ }^{t} G G g\right\rangle \geq(1-\eta)^{2}$ (by (3.4)), we have,

$$
\left\|h-\left\langle h, \chi_{V}\right\rangle \cdot \chi_{V}\right\|^{2}=\left\|h^{\perp}\right\|^{2} \leq 2 \eta / \gamma
$$

Now we bound

$$
\begin{aligned}
1-\max _{\varphi}\|G \varphi\|^{2} & \leq \underset{\left(v, v^{\prime}\right)}{\mathbf{E}}\left[1-Q\left(v, v^{\prime}\right)\right](\text { by }(3.3)) \\
& \leq \underset{\left(v, v^{\prime}\right)}{\mathbf{E}}\left[\left(1-Q\left(v, v^{\prime}\right)\right) 9\left\{\left(h(v)-\left\langle h, \chi_{V}\right\rangle \cdot \chi_{V}(v)\right)^{2}+\left(h\left(v^{\prime}\right)-\left\langle h, \chi_{V}\right\rangle \cdot \chi_{V}\left(v^{\prime}\right)\right)^{2}+h(v) h\left(v^{\prime}\right)\right\}\right] \\
& \leq 36 \eta / \gamma+9 \underset{\left(v, v^{\prime}\right)}{\mathbf{E}}\left[\left(1-Q\left(v, v^{\prime}\right)\right) h(v) h\left(v^{\prime}\right)\right] \\
& \leq 36 \eta / \gamma+18 \eta(\text { by }(3.4)),
\end{aligned}
$$

where the second inequality is by $1 \leq 9(a-\bar{a})^{2}+9\left(a^{\prime}-\bar{a}\right)^{2}+9 a a^{\prime}$ for $\bar{a} \geq 2 / 3$ (see Section 3.2 in Version v3 of [16] for the proof). This completes the proof.

\section{PCP and Inapproximability of Label Cover}

In this section, we prove the following theorem (first assuming Theorem 4.2).

Theorem 4.1 (Theorem 1.5 restated). For any $\delta>0$, there exists a constant $\sigma$ such that LabelCover( $1, \delta)$ over regular projection games with the alphabet size $\sigma=|\Sigma|$ is NP-hard.

Theorem 4.2. There exists a constant $\varepsilon>0$ such that LabelCover $(1,1-\varepsilon)$ over regular projection games with $|\Sigma| \leq 7$ is NP-hard.

Proof of Theorem 4.1. We show a polynomial-time reduction from LabelCover $(1,1-\varepsilon)$ to LabelCover( $1, \delta)$ (for all constants $\varepsilon, \delta>0$ ). Let $G$ be a LabelCover instance with vertex sets $U$ and $V$ and alphabet $\Sigma$. Our reduction produces a LabelCover instance $\widetilde{G}$ from $G$ satisfying: (1) If $\operatorname{val}(G)=1$, then $\operatorname{val}(\widetilde{G})=1$, and (2) if $\operatorname{val}(G) \leq 1-\varepsilon$, then $\operatorname{val}(\widetilde{G}) \leq \delta$. First, convert $G$ into a game $G^{\prime}$ such that the constraint graph of ${ }^{t} G^{\prime} G^{\prime}$ has a spectral gap $\gamma$ for some $\gamma>0$. (There is an easy way to do this; see Appendix A in Version v3 of [16].) Then, output the $k$-fold parallel repetition of $G^{\prime}$ for some $k=O(\log (1 / \delta) / \varepsilon)$ as $\widetilde{G}$. If the original instance has value 1 , the resulting instance also has value 1 by defining assignments $f^{k}: U^{k} \rightarrow \Sigma^{k}, g^{k}: V^{k} \rightarrow \Sigma^{k}$ to $\widetilde{G}$ as $f^{k}=(f, f, \ldots, f), g^{k}=(g, g, \ldots, g)$ where $f: U \rightarrow \Sigma, g:$ $V \rightarrow \Sigma$ are optimal assignments to $G$. If the original instance has value at most $1-\varepsilon$, then (the contrapositive of) Theorem 3.3 shows that the value of the resulting instance is at most $(1-\Omega(\varepsilon))^{k} \leq \delta$.

In the rest of this section, our goal is to prove Theorem 4.2 only assuming the PCP theorem. We prove Theorem 4.2 by a sequence of reductions starting from the PCP theorem to Max 3SAT to Max 3SAT- $B$ to Max E3SAT-5 to LabelCover $(1,1-\varepsilon)$ on regular projection games with $|\Sigma| \leq 7$.

The first reduction from the PCP theorem to Max 3SAT is called gap-introducing reduction. It is a reduction from SAT, the satisfiability problem, (as a decision problem) to Max 3SAT (as a promise problem). The reduction takes as an input an instance $I$ of SAT and produces an instance $T(I)$ of Max 3SAT. In addition, for some constant $\varepsilon>0$ which does not depend on $I$, the reduction satisfies: (1) If $I$ is satisfiable, then so is $T(I)$, and (2) if $I$ is not satisfiable, then any assignment cannot satisfy more than a $(1-\varepsilon)$-fraction of clauses of $T(I)$. 
The rest of reductions are called gap-preserving reduction. They are reductions from promise problems to promise problems and have similar structures, i.e., satisfy properties of the form: For any $\varepsilon>0$, there exists $\varepsilon^{\prime}>0$ such that (1) if $I$ is satisfiable, then so is $T(I)$, and (2) if any assignment cannot satisfy more than a $(1-\varepsilon)$-fraction of clauses of $I$, then any assignment cannot satisfy more than a $\left(1-\varepsilon^{\prime}\right)$-fraction of clauses of $T(I)$.

\subsection{The PCP theorem}

In this section, we describe the PCP theorem, which gives a new definition of the class NP in terms of Probabilistically Checkable Proofs (PCPs). Recall that we say a language $L \subseteq\{0,1\}^{*}$ is in NP if there exist a polynomial $p$ and a deterministic polynomial time Turing machine $V$ (called verifier) such that

- (Completeness) if $I \in L$, then there exists a "short" witness $w \in\{0,1\}^{p(I \mid)}$ such that $V(I, w)$ accepts, and

- (Soundness) if $I \notin L$, then for every string $w \in\{0,1\}^{p(|I|)}, V(I, w)$ rejects, where we denote by $|I|$ the length of $I$.

We define PCPs by considering a probabilistic modification of the definition of NP. We consider a probabilistic polynomial time Turing machine $V$ (also called verifier) that is given an input $I$ and "oracle access" to a witness string $\pi \in\{0,1\}^{*}$. We model the fact that $V$ is a probabilistic algorithm by assuming that $V$, in addition to the input $I$ and the witness $\pi$, takes an additional input $R$, that is a sequence of random bits. Then $V$ performs a deterministic computation based on $I, \pi$ and $R$. For fixed $I$ and $\pi$, when we say " $V^{\pi}(I)$ accepts" we mean "the event that $V$ accepts when we are given oracle access to witness $\pi$, input $I$, and a uniformly distributed random input $R$." When we refer to the "probability that $V^{\pi}(I)$ accepts," we take the probability over the choices of $R$.

Definition 4.3. Let $r: \mathbb{N} \rightarrow \mathbb{N}$ and $q: \mathbb{N} \rightarrow \mathbb{N}$ be some functions. A verifier is $(r(n), q(n))$-restricted if, for every input $I$ of length $n$ and for every $\pi, V^{\pi}(I)$ uses at most $r(|I|)$ random bits and reads at most $q(|I|)$ bits of $\pi$.

We define the class PCP $[r(n), q(n)]$ as follows.

Definition 4.4. A language $L$ is in $\mathrm{PCP}[r(n), q(n)]$ if there exists an $(r(n), q(n))$-restricted verifier $V$ such that

- (Completeness) if $I \in L$, then there is $\pi$ such that $V^{\pi}(I)$ accepts with probability 1 , and

- (Soundness) if $I \notin L$, then for every $\pi$ the probability that $V^{\pi}(I)$ accepts is at most $1 / 2$.

We abuse the notation to denote $\cup_{r(n) \in \mathrm{R}, q(n) \in \mathrm{Q}} \mathrm{PCP}[r(n), q(n)]$ by $\mathrm{PCP}[\mathrm{R}, \mathrm{Q}]$ for sets of functions $\mathrm{R}, \mathrm{Q}$.

It is clear from the definition that $\mathrm{PCP}[0, \operatorname{poly}(n)]=\mathrm{NP}, \mathrm{PCP}[\operatorname{poly}(n), 0]=$ coRP $($ coRP is the class of languages that can be accepted by one-sided error probabilistic polynomial time Turing machines, i.e., $I \in L$ is always accepted while $I \notin L$ is accepted with probability at most $1 / 2)$. We can also show that $\mathrm{PCP}[O(\log n), O(\log n)] \subseteq \mathrm{NP}$. One might wonder what is the minimum function $q(n)$ satisfying $\mathrm{NP} \subseteq \mathrm{PCP}[O(\log n), q(n)]$. The PCP theorem gives a suprising answer to this quesiton.

Theorem 4.5 (The PCP Theorem). NP $=\mathrm{PCP}[O(\log n), O(1)]$.

The theorem was proved in [3,4], motivated by a relation between PCP and approximation discovered in [22]. The actual proof relies on previous work, as well as on several new results. We remark that in the context of interactive proof systems, NEXP $=\operatorname{PCP}[\operatorname{poly}(n)$, poly $(n)]$ was shown [5] before the PCP theorem.

\subsection{The PCP theorem to Max 3SAT}

In the maximum satisfiability problem (Max SAT), the task is, given a CNF formula, i.e., a set of clauses, to find an assignment that maximizes the number of satisfied clauses, where a clause is a disjunction of literals and a literal is a Boolean variable or its negation. We say a CNF formula $I$ is satisfiable if there exists an assignment that satisfies all the clauses of $I$. Max SAT is one of the most fundamental NP-hard problems. In Max $k$ SAT, we pose a restriction that input instances are $k \mathrm{CNF}$ formulas, i.e., each clause contains at most $k$ literals.

The PCP Theorem implies the following.

Theorem 4.6. There exist a constant $\varepsilon>0$ and a polynomial time reduction $T$ from SAT to Max $3 S A T$ such that for every instance I of SAT,

- (Completeness) if I is satisfiable, then $T(I)$ is satisfiable, and

- (Soundness) if I is not satisfiable, then no assignment satisfies at least $(1-\varepsilon)$-fraction of clauses of $T(I)$.

Note that the above theorem means that there is no polynomial time $(1-\varepsilon)$-approximation algorithm for Max 3SAT unless $\mathrm{P}=\mathrm{NP}$. Our proof follows the presentation of [49].

Proof. By the PCP theorem, we have SAT $\in \mathrm{PCP}[O(\log n), q]$, where $q$ is a constant. Let $V$ be an $(O(\log n), q)$-restricted verifier for SAT. We describe a reduction $T$ from SAT to Max 3SAT.

Given an instance $I$ of SAT, we would like to simulate the possible computation of the verifier by a 3CNF Boolean formula $T(I)$. Recall that for each $R, V$ chooses $q$ positions $i_{1}^{(I, R)}, \ldots, i_{q}^{(I, R)} \in \mathbb{N}$ and a Boolean function $f_{(I, R)}:\{0,1\}^{q} \rightarrow$ $\{0,1\}$ and accepts if and only if $f_{(I, R)}\left(\pi\left(i_{1}^{(I, R)}\right), \ldots, \pi\left(i_{q}^{(I, R)}\right)\right)=1$.

To construct $T(I)$, we introduce Boolean variables $x_{1}, \ldots, x_{\ell}$, where $\ell$ is the length of the witness $\pi$. Our intention is that we would like to identify an assignment to $T(I)$ and a witness $\pi$ to $V$. For every random input $R$ for $V$, we introduce a set of clauses $\psi_{(I, R)}$ that represent the constraint $f_{(I, R)}\left(\pi\left(i_{1}^{(I, R)}\right), \ldots, \pi\left(i_{q}^{(I, R)}\right)\right)=1$. This can be done with a $q \mathrm{CNF}$ having at most $2^{q}$ clauses since we can represent any $q$-variable Boolean function as a $q \mathrm{CNF}$ formula. Then, we convert clauses of length $q$ to clauses of length 3 , which can be done by introducing additional variables, as in the standard 
reduction from $k$ SAT to 3 SAT (for example $\left(x_{3} \vee \bar{x}_{5} \vee \bar{x}_{7} \vee x_{11}\right)$ becomes $\left(x_{3} \vee \bar{x}_{5} \vee y\right) \wedge\left(\bar{y} \vee \bar{x}_{7} \vee x_{11}\right)$ and so on). Thus, this transformation creates a formula $\psi_{(I, R)}^{\prime}$ with at most $q \times 2^{q} 3 \mathrm{CNF}$ clauses for each $R$. Now we can define $T(I)$ as the union of clauses, $T(I)=\cup_{R} \psi_{(I, R)}^{\prime}$. Note that the length of each string $R$ is $r(|I|)=O(\log |I|)$, so the number of such strings is polynomial in $|I|$.

Let us now see the relation between the optimum of $T(I)$ as an instance of Max 3SAT and the question of whether $I$ is satisfiable or not.

If $I$ is satisfiable, then there is a witness $\pi$ such that $V^{\pi}(I)$ accepts for every $R$. Set $x_{i}=\pi(i)$ for $1 \leq i \leq \ell$, and set the auxiliary variables (introduced in the transformation from $q \mathrm{CNF}$ to $3 \mathrm{CNF}$ ) appropriately, then the assignment satisfies all the clauses, and $T(I)$ is satisfiable.

If $I$ is not satisfiable, then consider an arbitrary assignment to the variables $x_{i}$ and to the auxiliary variables, and consider the string $\pi$ where $\pi(i)$ is set equal to $x_{i}$. The witness $\pi$ makes the verifier reject for at least half of the $R \in\{0,1\}^{r(|I|)}$, and for each such $R$, at least one of the clauses of $\psi_{(I, R)}^{\prime}$ representing $f_{(I, R)}$ is not satisfied. Thus, at least a fraction $\varepsilon=\frac{1}{2} \frac{1}{q 2^{q}}$ of clauses are not satisfied.

\subsection{Max 3SAT to Max 3SAT with bounded occurrences}

In the Max 3SAT- $d$ problem we are given a 3CNF formula, where every variable only occurs in at most $d$ clauses. In this section, we prove the following.

Theorem 4.7. There exist constants $\varepsilon>0, d \in \mathbb{N}$ and a polynomial time reduction $T$ from Max 3SAT to Max 3SAT$(2 d+1)$ such that for every instance $\varphi$ of Max $3 S A T$,

- (Completeness) if $\varphi$ is satisfiable, then $T(\varphi)$ is satisfiable, and

- (Soundness) if no assignment satisfies at least $(1-\varepsilon)$-fraction of clauses of $\varphi$, then no assignment satisfies at least $(1-\varepsilon /(1+3 d))$-fraction of clauses of $T(\varphi)$, and

- (Bounded occurrence) $T(\varphi)$ is an instance of Max 3SAT- $(2 d+1)$.

Our proof again follows the presentation of [49].

Proof. For the reduction we will need expander graphs of the following type.

Definition 4.8 (Expander Graph). An undirected (unweighted) graph $G=(V, E)$ is a 1-expander if, for every subset $S \subseteq V,|S| \leq|V| / 2$, the number of edges having one endpoint in $S$ and one in $V \backslash S$ is at least $|S|$.

For our purposes, it will be acceptable for the expander graph to have multiple edges. We will use polynomial time constructible 1-expanders of constant degree $d$ of [36].

Let now $\varphi$ be an instance of Max 3SAT with $n$ variables $x_{1}, \ldots, x_{n}$ and $m$ clauses $C_{1}, \ldots, C_{m}$. For simplicity, we assume every clause contains exactly 3 literals.

For each variable $x_{i}$, let occ $_{i}$ be the number of occurrences of $x_{i}$, that is, the number of clauses that involve the literal $x_{i}$ or the literal $\bar{x}_{i}$. We write $x_{i} \in C_{j}$ if the literal $x_{i}$ or the literal $\bar{x}_{i}$ occurs in clause $C_{j}$. For each $i$, construct a 1-expander

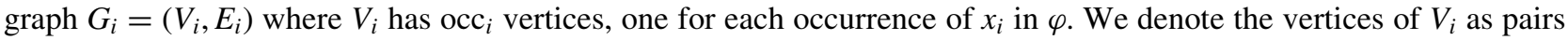
$[i, j]$ such that $x_{i}$ occurs in $C_{j}$. Each of these graphs has constant degree $d$.

We define a new instance $T(\varphi)$ of Max 3SAT with $N=3 m$ variables $Y=\left\{y_{i, j}\right\}_{i \in[n], C_{j} \ni x_{i}}$, one for each occurrence of each variable in $\varphi$. For each clause of $\varphi$ we put an equivalent clause in $T(\varphi)$. That is, if $C_{j}=\left(x_{a} \vee x_{b} \vee x_{c}\right)$ is a clause in $\varphi$, then $\left(y_{a, j} \vee y_{b, j} \vee y_{c, j}\right)$ is a clause in $T(\varphi)$. We call these clauses the primary clauses of $T(\varphi)$. Note that each variable of $T(\varphi)$ occurs only in one primary clause.

To complete the construction of $T(\varphi)$, for every variable $x_{i}$ in $\varphi$, and for every edge $\left([i, j],\left[i, j^{\prime}\right]\right)$ in the graph $G_{i}$, we add the clauses $\left(y_{i, j} \vee \bar{y}_{i, j^{\prime}}\right)$ and $\left(\bar{y}_{i, j} \vee y_{i, j^{\prime}}\right)$ to $T(\varphi)$. We call these clauses the consistency clauses of $T(\varphi)$. Note that if $y_{i, j}=y_{i, j^{\prime}}$ then both consistency clauses are satisfied, while if $y_{i, j} \neq y_{i, j^{\prime}}$ then one of the two consistency clauses is contradicted.

This completes the construction of $T(\varphi)$. By construction, every variable occurs in at most $2 d+1$ clauses of $T(\varphi)$, and $T(\varphi)$ has $M=m+3 d m$ clauses.

We now relate the optimum solutions in $T(\varphi)$ and $\varphi$.

Claim 4.9. If there is an assignment that satisfies all the clauses of $\varphi$, then there is an assignment that satisfies all the clauses of $T(\varphi)$.

Proof. Take the assignment for $\varphi$ and then for every variable $y_{i, j}$ of $T(\varphi)$ give the value that the assignment gives to $x_{i}$. This assignment satisfies all the consistency clauses and all the primary clauses.

Claim 4.10. If there is an assignment for $T(\varphi)$ that leaves at most $k$ clauses not satisfied, then there is an assignment for $\varphi$ that leaves at most $k$ clauses not satisfied.

Proof. Let $a_{i, j}$ be the value assigned to $y_{i, j}$. We first "round" the assignment so that all the consistency clauses are satisfied. This is done by defining an assignment $b_{i}$, where, for every $i$, the value $b_{i}$ is taken to be the majority value of $a_{i, j}$ over all $j$ such that $x_{i} \in C_{j}$, and we assign the value $b_{i}$ to all the variables $y_{i, j}$. The assignment $b_{i}$ satisfies all the consistency clauses, but it is possible that it contradicts some primary clauses that were satisfied by $a_{i, j}$. We claim that the $b_{i}$ assignment satisfies at least as many clauses as the $a_{i, j}$ assignment. Indeed, for each $i$, if $b_{i}$ differs from the $a_{i, j}$ for, 
say, $t$ values of $j$, then there can be at most $t$ primary clauses that were satisfied by $a_{i, j}$ but are contradicted by $b_{i}$. On the other hand, because of the consistency clauses being laid out as the edges of a 1-expander graph, at least $t$ consistency clauses are contradicted by the $a_{i, j}$ assignment, so the $b_{i}$ assignment can be no worse. We conclude that $b_{i}$ assignment contradicts no more clauses of $T(\varphi)$ than are contradicted by $a_{i, j}$, that is, no more than $k$ clauses. When we apply $b_{i}$ as an assignment for $\varphi$, we see that $b_{i}$ contradicts at most $k$ clauses of $\varphi$.

Setting $k=\varepsilon m=\varepsilon M /(1+3 d)$, we complete the proof.

\subsection{Max 3SAT with bounded occurrences to Max E3SAT-5 to LabelCover}

In the Max E3SAT-5 problem we are given a 3CNF formula with $n$ variables and $5 n / 3$ clauses, in which every clause contains exactly three literals, every variable appears in exactly five clauses, and a variable does not appear in a clause more than once.

In this section, we first prove the following.

Theorem 4.11. There exist a constant $\alpha=\alpha(d)>0$ and a polynomial time reduction T from Max 3SAT-d to Max E3SAT-5 such that for every instance $\varphi$ of Max 3SAT-d,

- (Completeness) if $\varphi$ is satisfiable, then $T(\varphi)$ is satisfiable, and

- (Soundness) if no assignment satisfies at least $(1-\varepsilon)$-fraction of clauses of $\varphi$, then no assignment satisfies at least $(1-\varepsilon \alpha(d))$-fraction of clauses of $T(\varphi)$, and

- (Bounded occurrence) $T(\varphi)$ is an instance of Max E3SAT-5.

Our proof follows the presentation of [21]. We only give a sketch of proof since it is similar to the proof of Theorem 4.7.

Proof. Let $\varphi$ be an instance of Max 3SAT- $d$ with $n$ variables $x_{1}, \ldots, x_{n}$ and $m$ clauses. For each variable $x_{i}$, let occ ${ }_{i}$ be the number of occurrences of $x_{i}$. For any $1 \leq i \leq n$, it holds that $\operatorname{occ}_{i} \leq d$, and without loss of generality, we also assume that $\operatorname{occ}_{i} \geq 2$.

We replace each occurrence of $x_{i}$ by a fresh variable. Let $x_{i, 1}, x_{i, 2}, \ldots, x_{i, \text { occ }_{i}}$ denote these fresh variables. For $1 \leq j \leq \operatorname{occ}_{i}$, we add the clauses $\left(x_{i, j} \vee \bar{x}_{i, j+1}\right),\left(\bar{x}_{i, j} \vee x_{i, j+1}\right)$, where $\operatorname{occ}_{i}+1$ is interpreted as 1 . These clauses are satisfied only if $x_{i, j}=x_{i, j+1}$ for every $j$. Now each variable appears exactly 5 times, and no variable appears more than once in the same clause.

For clauses that are shorter than three, we add one or two fresh literals to each of them so that they contain exactly three literals. Let $\bar{y}_{1}, \bar{y}_{2}, \ldots, \bar{y}_{\ell}$ denote these fresh literals. Note that $\ell$ is bounded from above by $2 m+\sum_{i} 2$ occ $_{i} \leq 4 m$. For each variable $y_{i}$, we introduce fresh variables $z_{i, 1}, z_{i, 2}$ and add the clauses $\left(y_{i} \vee z_{i, 1} \vee z_{i, 2}\right),\left(y_{i} \vee \bar{z}_{i, 1} \vee z_{i, 2}\right)$, $\left(y_{i} \vee z_{i, 1} \vee \bar{z}_{i, 2}\right),\left(y_{i} \vee \bar{z}_{i, 1} \vee \bar{z}_{i, 2}\right)$. These clauses are satisfied only if $y_{i}=1$, in which case $\bar{y}_{i}$ has no influence on the original clause to which it was added.

Now each variable of $\left\{y_{i}\right\}$ and $\left\{z_{i, j}\right\}$ appears exactly 5 and 4 times respectively. Let $t$ be the number of the variables of type $z_{i, j}$. If $t \equiv 1(\bmod 3)$, we introduce a fresh variable $w_{1}$. If $t \equiv 2(\bmod 3)$, we introduce fresh variables $w_{1}, w_{2}$. Then, we add clauses that contain distinct variables $z_{i, j}$ and $w_{i}$ in positive form, until each variable occurs exactly five times (we use each variable of type $w_{i}$ five times).

This completes the construction of $T(\varphi)$. By construction, every variable occurs exactly in 5 clauses of $T(\varphi)$, and $T(\varphi)$ has at most $M=m+2 d n+4(2 m+2 d n)+\lfloor 4(2 m+2 d n) / 3\rfloor+4=O(d m)$ clauses.

We can prove the following similarly as before.

Claim 4.12. If there is an assignment that satisfies all the clauses of $\varphi$, then there is an assignment that satisfies all the clauses of $T(\varphi)$.

Claim 4.13. If there is an assignment for $T(\varphi)$ that leaves at most $k$ clauses not satisfied, then there is an assignment for $\varphi$ that leaves at most $k$ clauses not satisfied.

We complete the proof.

Now we are ready to prove Theorem 4.2.

Proof of Theorem 4.2. We construct a regular projection game $G=(U, V, E, \Sigma, W, \Pi)$ from an instance $\varphi$ of Max E3SAT-5 with variables $x_{1}, x_{2}, \ldots, x_{n}$ and clauses $C_{1}, C_{2}, \ldots, C_{m}$ as follows: Define $U:=\left\{u_{1}, u_{2}, \ldots, u_{n}\right\}, V:=$ $\left\{v_{1}, v_{2}, \ldots, v_{m}\right\}, E:=\left\{\left(u_{i}, v_{j}\right) \mid x_{i} \in C_{j}\right\}, \Sigma:=\{1,2, \ldots, 7\}$ and $w_{e}=1$ for any $w_{e} \in W$. We associate the elements of $\{0,1\}$ and $\{0,1\}^{3} \backslash\{(0,0,0)\}$ with the elements of $\Sigma$ as shown in Table 2.

For $e=\left(u_{i}, v_{j}\right) \in E$, a constraint $\pi_{e} \in \Pi$ is satisfied if $x_{i}$ and $C_{j}$ have consistent labels. For example, let $C_{1}=$ $\left(x_{1} \vee \bar{x}_{2} \vee x_{3}\right)$ and consider an edge $e=\left(u_{2}, v_{1}\right) . \pi_{e}$ is satisfied if $u_{2}$ and $v_{1}$ are assigned 1 and 2 respectively because $x_{2}$ and $C_{1}$ are assigned $0 \in\{0,1\}$ and $(0,1,0) \in\{0,1\}^{3} \backslash\{(0,0,0)\}$ respectively, and thus, $x_{2}=0$ and $\left(x_{1}=0, \bar{x}_{2}=1\right.$, $\left.x_{3}=0\right)$ are consistent. $\pi_{\left(u_{1}, v_{1}\right)}, \pi_{\left(u_{2}, v_{1}\right)}, \pi_{\left(u_{3}, v_{1}\right)}$ are explicitly described as follows.

$$
\begin{aligned}
& \pi_{\left(u_{1}, v_{1}\right)}=\{(1,1),(1,2),(1,3),(2,4),(2,5),(2,6),(2,7)\}, \\
& \pi_{\left(u_{2}, v_{1}\right)}=\{(1,2),(1,3),(1,6),(1,7),(2,1),(2,4),(2,5)\},
\end{aligned}
$$


Table 2. Associating elements

\begin{tabular}{ccc}
\hline$\Sigma$ & $\{0,1\}$ & $\{0,1\}^{3} \backslash\{(0,0,0)\}$ \\
\hline 1 & 0 & $(0,0,1)$ \\
2 & 1 & $(0,1,0)$ \\
3 & - & $(0,1,1)$ \\
4 & - & $(1,0,0)$ \\
5 & - & $(1,0,1)$ \\
6 & - & $(1,1,0)$ \\
7 & - & $(1,1,1)$ \\
\hline
\end{tabular}

$$
\pi_{\left(u_{3}, v_{1}\right)}=\{(1,2),(1,4),(1,6),(2,1),(2,3),(2,5),(2,7)\} .
$$

We can easily verify the following claims.

- (Completeness) if $\varphi$ is satisfiable, then $\operatorname{val}(G)=1$, and

- (Soundness) if no assignment satisfies at least $(1-\varepsilon)$-fraction of clauses of $\varphi$, then $\operatorname{val}(G) \leq(1-\varepsilon / 3)$, and

- (Regular and projection) $G$ is a regular projection game.

This completes the proof.

\section{Concluding Remarks}

In this paper, we present a proof of the parallel repetition theorem following the matrix analysis approach of Dinur and Steurer [17]. There are many related topics we cannot mention in this paper such as direct sum and direct product in communication complexity $[6,11,40]$, entangled games and EPR paradox $[14,29,30]$, foams and tiling the space $\mathbb{R}^{n}$ $[1,23,33]$, to name a few. A brief overview of such topics can be found in [43].

\section{Acknowledgments}

We would like to thank two reviewers for their careful reading and helpful comments on our paper. We would like to express the deepest appreciation to Akiyoshi Shioura for his great help with improving the presentation of the paper.

\section{REFERENCES}

[1] N. Alon and B. Klartag. Economical toric spines via Cheeger's inequality. Journal of Topology and Analysis, 1(2):101-111, 2009.

[2] S. Arora and B. Barak. Computational Complexity - A Modern Approach. Cambridge University Press, 2009.

[3] S. Arora, C. Lund, R. Motwani, M. Sudan, and M. Szegedy. Proof verification and the hardness of approximation problems. J. ACM, 45(3):501-555, 1998.

[4] S. Arora and S. Safra. Probabilistic checking of proofs: A new characterization of NP. J. ACM, 45(1):70-122, 1998.

[5] L. Babai, L. Fortnow, and C. Lund. Non-deterministic exponential time has two-prover interactive protocols. Computational Complexity, 1:3-40, 1991.

[6] B. Barak, M. Braverman, X. Chen, and A. Rao. How to compress interactive communication. SIAM J. Comput., 42(3):13271363, 2013.

[7] B. Barak, M. Hardt, I. Haviv, A. Rao, O. Regev, and D. Steurer. Rounding parallel repetitions of unique games. In Proceedings of the 49th Annual IEEE Symposium on Foundations of Computer Science (FOCS), pages 374-383, 2008.

[8] B. Barak, A. Rao, R. Raz, R. Rosen, and R. Shaltiel. Strong parallel repetition theorem for free projection games. In Proceedings of APPROX-RANDOM 2009, pages 352-365, 2009.

[9] M. Ben-Or, S. Goldwasser, J. Kilian, and A. Wigderson. Multi-prover interactive proofs: How to remove intractability assumptions. In Proceedings of the 20th Annual ACM Symposium on Theory of Computing (STOC), pages 113-131, 1988.

[10] M. Ben-Or, S. Goldwasser, J. Kilian, and A. Wigderson. Efficient identification schemes using two prover interactive proofs. In Proceedings of the 9th Annual International Cryptology Conference (CRYPTO), pages 498-506, 1989.

[11] M. Braverman, A. Rao, O. Weinstein, and A. Yehudayoff. Direct products in communication complexity. In Proceedings of the 54th Annual IEEE Symposium on Foundations of Computer Science (FOCS), pages 746-755, 2013.

[12] J.-Y. Cai, A. Condon, and R. J. Lipton. On games of incomplete information. Theor. Comput. Sci., 103(1):25-38, 1992.

[13] A. Chailloux and G. Scarpa. Parallel repetition of entangled games with exponential decay via the superposed information cost. In Proceedings of the 41st International Colloquium on Automata, Languages, and Programming (ICALP) Part I, pages 296-307, 2014.

[14] R. Cleve, W. Slofstra, F. Unger, and S. Upadhyay. Perfect parallel repetition theorem for quantum xor proof systems. Computational Complexity, 17(2):282-299, 2008.

[15] I. Dinur and P. Harsha. Composition of low-error 2-query PCPs using decodable PCPs. In Proceedings of the 50th Annual IEEE Symposium on Foundations of Computer Science (FOCS), pages 472-481, 2009.

[16] I. Dinur and D. Steurer. Analytical approach to parallel repetition. CoRR, abs/1305.1979, 2013.

[17] I. Dinur and D. Steurer. Analytical approach to parallel repetition. In Proceedings of the 46th Annual ACM Symposium on 
Theory of Computing (STOC), pages 624-633, 2014.

[18] I. Dinur, D. Steurer, and T. Vidick. A parallel repetition theorem for entangled projection games. In Proceedings of the 29th Annual IEEE Conference on Computational Complexity (CCC), pages 197-208, 2014.

[19] C. Dwork, U. Feige, J. Kilian, M. Naor, and S. Safra. Low communication 2-prover zero-knowledge proofs for NP. In Proceedings of the 12th Annual International Cryptology Conference (CRYPTO), pages 215-227, 1992.

[20] U. Feige. On the success probability of the two provers in one-round proof systems. In Proceedings of the 6th Annual Structure in Complexity Theory Conference, pages 116-123, 1991.

[21] U. Feige. A threshold of $\ln n$ for approximating set cover. J. ACM, 45(4):634-652, 1998.

[22] U. Feige, S. Goldwasser, L. Lovász, S. Safra, and M. Szegedy. Interactive proofs and the hardness of approximating cliques. J. ACM, 43(2):268-292, 1996.

[23] U. Feige, G. Kindler, and R. O'Donnell. Understanding parallel repetition requires understanding foams. In Proceedings of the 22nd Annual IEEE Conference on Computational Complexity (CCC), pages 179-192, 2007.

[24] U. Feige and L. Lovász. Two-prover one-round proof systems: Their power and their problems (extended abstract). In Proceedings of the 24th Annual ACM Symposium on Theory of Computing (STOC), pages 733-744, 1992.

[25] J. Håstad. Clique is hard to approximate within $n^{1-\varepsilon}$. Acta Mathematica, 182(1):105-142, 1999.

[26] J. Håstad. Some optimal inapproximability results. J. ACM, 48(4):798-859, 2001.

[27] T. Holenstein. Parallel repetition: Simplification and the no-signaling case. Theory of Computing, 5(1):141-172, 2009.

[28] R. Jain, A. Pereszlényi, and P. Yao. A parallel repetition theorem for entangled two-player one-round games under product distributions. In Proceedings of the 29th Annual IEEE Conference on Computational Complexity (CCC), pages 209-216, 2014.

[29] J. Kempe and O. Regev. No strong parallel repetition with entangled and non-signaling provers. In Proceedings of the 25th Annual IEEE Conference on Computational Complexity (CCC), pages 7-15, 2010.

[30] J. Kempe and T. Vidick. Parallel repetition of entangled games. In Proceedings of the 43rd ACM Symposium on Theory of Computing (STOC), pages 353-362, 2011.

[31] S. Khot. On the power of unique 2-prover 1-round games. In Proceedings of the 34th Annual ACM Symposium on Theory of Computing (STOC), pages 767-775, 2002.

[32] S. Khot. On the unique games conjecture (invited survey). In Proceedings of the 25th Annual IEEE Conference on Computational Complexity (CCC), pages 99-121, 2010.

[33] G. Kindler, A. Rao, R. O'Donnell, and A. Wigderson. Spherical cubes: Optimal foams from computational hardness amplification. Commun. ACM, 55(10):90-97, 2012.

[34] D. Lapidot and A. Shamir. A one-round, two-prover, zero-knowledge protocol for NP. Combinatorica, 15(2):204-214, 1995.

[35] D. Lapidot and A. Shamir. Fully parallelized multi-prover protocols for NEXP-time. J. Comput. Syst. Sci., 54(2):215-220, 1997.

[36] A. Lubotzky, R. Phillips, and P. Sarnak. Ramanujan graphs. Combinatorica, 8(3):261-277, 1988.

[37] C. Lund, L. Fortnow, H. J. Karloff, and N. Nisan. Algebraic methods for interactive proof systems. J. ACM, 39(4):859-868, 1992.

[38] C. Lund and M. Yannakakis. On the hardness of approximating minimization problems. J. ACM, 41(5):960-981, 1994.

[39] D. Moshkovitz and R. Raz. Two-query PCP with subconstant error. J. ACM, 57(5), 2010.

[40] I. Parnafes, R. Raz, and A. Wigderson. Direct product results and the GCD problem, in old and new communication models. In Proceedings of the Twenty-Ninth Annual ACM Symposium on the Theory of Computing (STOC), pages 363-372, 1997.

[41] A. Rao. Parallel repetition in projection games and a concentration bound. SIAM J. Comput., 40(6):1871-1891, 2011.

[42] R. Raz. A parallel repetition theorem. SIAM J. Comput., 27(3):763-803, 1998.

[43] R. Raz. Parallel repetition of two prover games (invited survey). In Proceedings of the 25th Annual IEEE Conference on Computational Complexity, pages 3-6, 2010

[44] R. Raz. A counterexample to strong parallel repetition. SIAM J. Comput., 40(3):771-777, 2011.

[45] R. Raz and R. Rosen. A strong parallel repetition theorem for projection games on expanders. In Proceedings of the 27th Conference on Computational Complexity (CCC), pages 247-257, 2012.

[46] A. Shamir. IP = PSPACE. J. ACM, 39(4):869-877, 1992.

[47] D. Steurer. Improved rounding for parallel repeated unique games. In Proceedings of APPROX-RANDOM 2010, pages 724737,2010

[48] D. Steurer. On the Complexity of Unique Games and Graph Expansion. PhD thesis, Princeton University, 2010.

[49] L. Trevisan. Inapproximability of combinatorial optimization problems. Manuscript.

[50] S. P. Vadhan. Pseudorandomness. Foundations and Trends in Theoretical Computer Science, 7(1-3):1-336, 2012.

[51] O. Verbitsky. Towards the parallel repetition conjecture. Theor. Comput. Sci., 157(2):277-282, 1996. 